\title{
NUMERICAL APPROXIMATION OF A VARIATIONAL INEQUALITY RELATED TO THE HUMID ATMOSPHERE*
}

\author{
ROGER TEMAM ${ }^{\dagger}$ AND XIAOYAN WANG TE $^{\ddagger}$
}

\begin{abstract}
We investigate the numerical approximation of solutions to some variational inequalities modeling the humid atmosphere when the saturation of water vapor in the air is taken into account. In order to overcome the difficulties caused by the constraints on the humidity $q$ $(0 \leq q \leq 1)$ and the discontinuity in the variational inequalities, we construct a penalized and regularized implicit Euler method. We manage to show that the approximation functions associated with the numerical scheme converge to the solutions of the variational inequalities through deriving various delicate a priori estimates and by using compactness arguments.
\end{abstract}

Key words. atmosphere equation, variational inequality, penalization, regularization, uniform estimates

AMS subject classifications. 35Q35, 65M06, 65M12, 35B30, 76D03, 86A10

DOI. $10.1137 / 15 \mathrm{M} 1039420$

1. Introduction. It is known that our lack of understanding of the physics of the clouds is a major cause of uncertainty in current weather predictions. Therefore, the investigation of the humid atmosphere is an important step towards a better understanding of weather predictions in a short time period and climate changes in a long time period. The primitive equations are the classical tool used in the study of climate and weather predictions; they describe the motion of the atmosphere when the hydrostatic assumption is enforced (see [17, 18, 22]). The rigorous mathematical theory of the equations of the humid atmosphere has been initiated in [21] and has attracted the attention of a number of practitioners from different fields; see, e.g., $[3,8,11,13,14,23,24]$ and the references therein.

The original primitive equations of the atmosphere are a system of nonlinear partial differential equations on the temperature variable $T$, vapor humidity $q$, and the velocity field $\mathbf{u}$, which are all unknown functions of the spatial and temporal variables. The studies of the humid atmosphere in, e.g., [21] and, more recently, $[15,16]$ do not account for an important phenomenon, namely the saturation of water vapor in the air, so that the equation for the vapor humidity $q$ appears as a simple transport equation. The equations for the saturated atmosphere appear, for example, in $[17,18,25]$. They involve $T, q$, and $\mathbf{u}$ and the saturation concentration $q_{s}$. In the first studies $[1,4,28]$ the saturation concentration $q_{s}$ is assumed to be constant. In $[1,4,28]$ the velocity is supposed to be given, and the system only involves $T$ and $q$, and in [5] the velocity $\mathbf{u}$ is also unknown. These articles provide a formulation of the equations of the humid atmosphere which involve thresholds (condensation and evaporation) and are thus nonlinear and noncontinuous (and nonmonotone). They also provide results of existence, uniqueness, and regularity of solutions.

* Received by the editors September 15, 2015; accepted for publication (in revised form) October 10, 2016; published electronically February 8, 2017.

http://www.siam.org/journals/sinum/55-1/M103942.html

Funding: This work was partially supported by the National Science Foundation under grants DMS-1206438 and DMS-1510249 and by the Research Fund of Indiana University.

${ }^{\dagger}$ Institute for Scientific Computing and Applied Mathematics, Indiana University, Bloomington, IN 47405 (temam@indiana.edu).

${ }^{\ddagger}$ Corresponding author. Institute for Scientific Computing and Applied Mathematics, Indiana University, Bloomington, IN 47405 (wang264@indiana.edu). 
In trying to extend the study to the case where $q_{s}$ is not constant [29], it was found that the equations of the humid atmosphere in $[17,18,25]$ are not coherent in the extreme cases where $q=0$ and $q=1(0 \leq q \leq 1$ by definition of $q)$, whether $q_{s}$ is constant or not; $q=0$ corresponds to a totally dry atmosphere, and $q=1$ corresponds to a totally humid atmosphere. A physically satisfactory resolution of this difficulty is proposed in [29] and studied mathematically in [31]; namely, the equation for $q$ is replaced by a variational inequality. The natural convex set for this problem is $H^{1}(\mathcal{M}) \times \mathcal{K}, T \in H^{1}(\mathcal{M}), q \in \mathcal{K}=\left\{\tilde{q} \in H^{1}(\mathcal{M}), 0 \leq \tilde{q} \leq 1\right.$ a.e. $\}$. The utilization of variational inequalities for the study of unilateral problems in mechanics and physics is the object of a vast literature; see, e.g., [2, 9, 19].

Our aim in this article is to study the numerical approximation of the variational inequalities introduced in $[29,31]$, for simplicity in the case where $q_{s}$ is constant; the case where $q_{s}$ is not constant will be addressed elsewhere [30], but it does not induce major additional mathematical difficulties. In the current paper, we shall follow the former works (see $[1,4,28]$ ) and assume, for the sake of simplicity, that the velocity $\mathbf{u}$ of the humid air is known. We believe that despite these simplifications, the resulting system contains the essence of the nonlinearity that is present in the moist advection. See section 2 for a more precise presentation of the model under investigation. The existence of change in phases leads to the introduction of a setvalued Heaviside function. More equations containing the Heaviside function can be found in, e.g., $[6,7,10,12]$. The introduction of such a set-valued function makes the equations for the vapor humidity $q$ and temperature $T$ nonlinear, discontinuous, and nonmonotone.

We address in the current paper the numerical approximation of the solutions to the variational inequalities derived from the humidity equations or the primitive equations. As we have pointed out in the previous paragraph, the problems we study here contain discontinuities and involve inequalities. They are the two distinct features of the model that we study. The discontinuities and variational inequalities which come from the changes of phases and the extreme cases for the vapor concentration bring significant mathematical difficulties to the understanding of the model. In the current work, we propose an implicit Euler scheme to approach the solutions to the system. However, we cannot simply proceed from this scheme directly as usual due to the difficulties induced from the discontinuities and physical requirement for the vapor concentration $q$. More precisely, we have to introduce a regularized version of the scheme to overcome the difficulties caused by the discontinuities in the model. Meanwhile, the unknown function $q$ should satisfy the range condition $0 \leq q \leq 1$ a.e. in the underlying domain which is denoted by $\mathcal{M}$ below. As the initial discretization in the usual Euler scheme cannot guarantee that the functions we recursively define obey this range condition, we have used a penalization technique in the regularized Euler scheme to achieve this range condition in the limit through delicate energy estimates. In summary, we discretize the variational inequality using an implicit Euler scheme, and we use penalization and regularization to show the existence of solutions to the Euler scheme. Then to prove convergence of the Euler scheme, we classically need some strong convergence results which follow from additional a priori estimates on the discretized Euler scheme. Obtaining a priori estimates for the time derivatives of solutions to evolutionary inequalities was stated as an important open problem in [20]. Though this problem has been solved in the general setting in [2], the abstract setting of [2] does not directly apply in our current setting, and we directly obtain the desired estimates by a delicate analysis of the penalized terms. Our main result is Theorem 4.4 . 
The rest of the article is organized as follows. In section 2, we give a precise formulation of the problem. In section 3, we introduce the Euler scheme and derive various uniform estimates for the functions associated with the penalized and regularized scheme. In section 4, we investigate the convergence of the Euler scheme.

\section{The exact problem: Formulation and setting.}

2.1. Formulation of the exact problem. Let $\mathcal{M}=\mathcal{M}^{\prime} \times\left(p_{0}, p_{1}\right)$, where $\mathcal{M}^{\prime} \subset$ $\mathbb{R}^{2}$, be a bounded domain with smooth boundary, and let $p_{0}, p_{1}$ be two positive numbers such that $p_{0}<p_{1}$. A typical point in $\mathcal{M}$ is $(x, y, p) ; \mathbf{u}:=(\mathbf{v}(x, y, p, t), \omega(x, y, p, t))$ $\in \mathbb{R}^{3}$ is a given datum which is the velocity of the fluid; $\nabla=\left(\partial_{x}, \partial_{y}\right)$ and $\Delta=\partial_{x}^{2}+\partial_{y}^{2}$ are the horizontal gradient and horizontal Laplace operators, respectively. The unknown in our problem will be $q$, the specific humidity, and $T$, the temperature. We denote by $q_{s}$ the saturation specific humidity which does not depend on the time variable $t$. The boundary $\partial \mathcal{M}$ of the domain $\mathcal{M}$ is decomposed as $\partial \mathcal{M}=\Gamma_{i} \cup \Gamma_{u} \cup \Gamma_{l}$, corresponding, respectively, to the bottom, top, and lateral boundaries of $\mathcal{M}$.

Let $t_{1}>0$ be a fixed constant, and let $\mathcal{K}$ be the nonempty closed convex set in $H^{1}(\mathcal{M})$ defined as $\mathcal{K}=\left\{q \in H^{1}(\mathcal{M}) ; 0 \leq q \leq 1\right.$ a.e. $\}$. Our problem is formulated as follows:

Find $T:\left(0, t_{1}\right) \rightarrow H^{1}(\mathcal{M}), q:\left(0, t_{1}\right) \rightarrow \mathcal{K}$, and $h_{q} \in \mathcal{H}\left(q-q_{s}\right)$ such that for any $q^{b} \in \mathcal{K}$ and $t \in\left(0, t_{1}\right)$, there hold

$$
\begin{gathered}
\partial_{t} T+\mathcal{A}_{T} T+\mathbf{v} \cdot \nabla T+\omega \partial_{p} T-\frac{R \omega}{c_{p} p} T=\frac{1}{p} \omega^{-} h_{q} \varphi(T), \\
\left\langle\partial_{t} q, q^{b}-q\right\rangle+\left(\mathcal{A}_{q} q+\mathbf{v} \cdot \nabla q+\omega \partial_{p} q, q^{b}-q\right) \geq\left(-\frac{1}{p} \omega^{-} h_{q} F(T), q^{b}-q\right),
\end{gathered}
$$

with the boundary and initial conditions

$$
\begin{aligned}
& \partial_{p} T=\alpha\left(T_{*}-T\right), \partial_{p} q=\beta\left(q_{*}-q\right) \text { on } \Gamma_{i} ; \partial_{p} T=0, \partial_{p} q=0 \text { on } \Gamma_{u} ; \\
& \partial_{n} T=0, \partial_{n} q=0 \text { on } \Gamma_{l}, \text { where } n \text { is the outward normal vector to } \Gamma_{l} ; \\
& T(x, y, p, 0)=T_{0}(x, y, p), q(x, y, z, 0)=q_{0}(x, y, p) .
\end{aligned}
$$

In the above, $\mathcal{H}$ is the set-valued Heaviside function $\mathcal{H}(\cdot)$ such that $\mathcal{H}(0)=[0,1]$. We decompose a function as $f=f^{+}-f^{-}$, where $f^{+}:=\max \{f, 0\}$ and $f^{-}=\max \{-f, 0\}$.

Throughout the paper, we shall assume the boundary data $T_{*}$ and $q_{*}$ to satisfy

$$
T_{*}, q_{*} \in L^{2}\left(0, t_{1} ; L^{2}\left(\Gamma_{i}\right)\right) .
$$

Naturally, we shall assume $q_{0} \in H^{1}(\mathcal{M}), q_{0} \in \mathcal{K}$, and $0 \leq q_{*} \leq 1$. The operators $\mathcal{A}_{T}$ and $\mathcal{A}_{q}$ are defined as

$$
\mathcal{A}_{T}=-\mu_{1} \Delta-\nu_{1} \partial_{p}\left(\left(\frac{g p}{R \bar{T}}\right)^{2} \partial_{p}\right), \quad \mathcal{A}_{q}=-\mu_{2} \Delta-\nu_{2} \partial_{p}\left(\left(\frac{g p}{R \bar{T}}\right)^{2} \partial_{p}\right),
$$

where $\mu_{i}, \nu_{i}, g, R, c_{p}$ are positive constants, $\bar{T}=\bar{T}(p)$ is the average temperature over the isobar with pressure $p$, and we assume that

$$
\bar{T}_{*} \leq \bar{T}(p) \leq \bar{T}^{*}, \quad\left|\partial_{p} \bar{T}(p)\right| \leq M \quad \text { for some positive constants } \bar{T}_{*}, \bar{T}^{*}, M .
$$

The functions $F$ and $\varphi$, both from $\mathbb{R}^{1}$ to $\mathbb{R}^{1}$, are defined as (see $[17,18,25]$ )

$$
F(\zeta)=q_{s} \zeta \frac{R L(\zeta)-c_{p} R_{v} \zeta}{c_{p} R_{v} \zeta^{2}+q_{s} L(\zeta)^{2}}, \text { with } L(\zeta)=c_{1}-c_{2} \zeta ; \varphi(\zeta)=\frac{1}{c_{p}} L(\zeta) F(\zeta) .
$$


Above, $c_{1}, c_{2}, R, R_{v}, R_{q}$ are all strictly positive constants; see, e.g., [4, 17, 18, 25] for more details on these constants.

It is easy to see that the rational function $F$ is bounded and globally Lipschitz on $\mathbb{R}^{1}$, i.e.,

$$
|F(\zeta)| \leq C<+\infty, \quad\left|F\left(\zeta_{1}\right)-F\left(\zeta_{2}\right)\right| \leq C\left|\zeta_{1}-\zeta_{2}\right| \quad \forall \zeta, \zeta_{1}, \zeta_{2} \in \mathbb{R}^{1}
$$

The function $\varphi$ is also globally Lipschitz:

$$
\left|\varphi\left(\zeta_{1}\right)-\varphi\left(\zeta_{2}\right)\right| \leq C\left|\zeta_{1}-\zeta_{2}\right| \quad \forall \zeta_{1}, \zeta_{2} \in \mathbb{R}^{1}
$$

In addition, $\varphi(0)=0$. Therefore, the Lipschitz function $\varphi$ has a sublinear estimate $|\varphi(\zeta)| \leq C|\zeta|$ for any $\zeta \in \mathbb{R}^{1}$.

2.2. Functional formulation. We denote as usual $H=L^{2}(\mathcal{M}), V=H^{1}(\mathcal{M})$. We use $(\cdot, \cdot)_{L^{2}}$ (regarded the same as $\left.(\cdot, \cdot)_{H}\right)$ and $|\cdot|_{L^{2}}$ to denote the usual scalar product and induced norm in $H$. In the space $V$, we will use $((\cdot, \cdot))$ and $\|\cdot\|$ to denote the scalar product adapted to the problem under investigation,

$$
((\varphi, \phi)):=(\nabla \varphi, \nabla \phi)+\left(\partial_{p} \varphi, \partial_{p} \phi\right)+\int_{\Gamma_{i}} \varphi \phi d \Gamma_{i}
$$

and the corresponding norm. The symbol $\langle\cdot, \cdot\rangle$ will denote the duality pairing between a Banach space $E$ and its dual space $E^{*}$. Associated with the Navier-Stokes equations, we also use the following standard notation:

$$
\begin{aligned}
& \mathbf{H}=\{\mathbf{u} \in H \times H \times H \mid \operatorname{div} \mathbf{u}=0 \text { and } \mathbf{u} \cdot n=0 \text { on } \partial \mathcal{M}\} \\
& \mathbf{V}=\{\mathbf{u} \in V \times V \times V \mid \operatorname{div} \mathbf{u}=0 \text { and } \mathbf{u} \cdot n=0 \text { on } \partial \mathcal{M}\}
\end{aligned}
$$

which will serve as the natural function space for the vector field $\mathbf{u}$.

For $T, T^{b}, q, q^{b} \in V$, we see through integration by parts and using the Neumann boundary conditions that

$$
\begin{aligned}
\left\langle\mathcal{A}_{T} T, T^{b}\right\rangle & =a_{T}\left(T, T^{b}\right)-\nu_{1} \alpha \int_{\Gamma_{i}}\left(\frac{g p_{1}}{R \bar{T}}\right)^{2} T_{*} T^{b} d \Gamma_{i}, \\
\left\langle\mathcal{A}_{q} q, q^{b}\right\rangle & =a_{q}\left(q, q^{b}\right)-\nu_{2} \beta \int_{\Gamma_{i}}\left(\frac{g p_{1}}{R \bar{T}}\right)^{2} q_{*} q^{b} d \Gamma_{i},
\end{aligned}
$$

where the bilinear forms $a_{T}\left(T, T^{b}\right), a_{q}\left(q, q^{b}\right)$ are defined by

$a_{T}\left(T, T^{b}\right)=\mu_{1}\left(\nabla T, \nabla T^{b}\right)_{H}+\nu_{1} \int_{\mathcal{M}}\left(\frac{g p}{R \bar{T}}\right)^{2} \partial_{p} T \partial_{p} T^{b} d \mathcal{M}+\nu_{1} \alpha \int_{\Gamma_{i}}\left(\frac{g p_{1}}{R \bar{T}}\right)^{2} T T^{b} d \Gamma_{i}$,

$$
a_{q}\left(q, q^{b}\right)=\mu_{2}\left(\nabla q, \nabla q^{b}\right)_{H}+\nu_{2} \int_{\mathcal{M}}\left(\frac{g p}{R \bar{T}}\right)^{2} \partial_{p} q \partial_{p} q^{b} d \mathcal{M}+\nu_{2} \beta \int_{\Gamma_{i}}\left(\frac{g p_{1}}{R \bar{T}}\right)^{2} q q^{b} d \Gamma_{i} .
$$

Copyright (c) by SIAM. Unauthorized reproduction of this article is prohibited. 
We also set $U:=(T, q), U^{b}:=\left(T^{b}, q^{b}\right)$ and define

$b_{T}\left(\mathbf{u}, T, T^{b}\right)=\int_{\mathcal{M}}\left(\mathbf{v} \cdot \nabla T+\omega \partial_{p} T\right) T^{b} d \mathcal{M}$ and $b_{q}\left(\mathbf{u}, q, q^{b}\right)=\int_{\mathcal{M}}\left(\mathbf{v} \cdot \nabla q+\omega \partial_{p} q\right) q^{b} d \mathcal{M}$,

$$
l\left(U^{b}\right):=l_{T}\left(T^{b}\right)+l_{q}\left(q^{b}\right)=\nu_{1} \alpha \int_{\Gamma_{i}}\left(\frac{g p_{1}}{R \bar{T}}\right)^{2} T_{*} T^{b} d \Gamma_{i}+\nu_{2} \beta \int_{\Gamma_{i}}\left(\frac{g p_{1}}{R \bar{T}}\right)^{2} q_{*} q^{b} d \Gamma_{i} .
$$

We then introduce the operators associated with these forms, namely, $A_{T}, A_{q}$ : $A_{T}, A_{q}: V \rightarrow V^{*}$ defined by

$$
\left\langle A_{T} u, v\right\rangle:=a_{T}(u, v), \quad\left\langle A_{q} u, v\right\rangle:=a_{q}(u, v), \quad \forall u, v \in V .
$$

Similarly, we define $B(\mathbf{u}, U)=\left(B_{T}(\mathbf{u}, U), B_{q}(\mathbf{u}, q)\right): \mathbf{V} \times V^{2} \rightarrow\left(V^{*}\right)^{2}$ (with $\left.V^{2}=V \times V,\left(V^{*}\right)^{2}=V^{*} \times V^{*}\right)$ and $D(\mathbf{u}, u): \mathbf{H} \times V \rightarrow V^{\prime}$ by setting

$$
\left\langle B(\mathbf{u}, U), U^{b}\right\rangle:=\left(b_{T}\left(\mathbf{u}, T, T^{b}\right), b_{q}\left(\mathbf{u}, q, q^{b}\right)\right) \quad \forall \mathbf{u} \in \mathbf{V}, U, U^{b} \in V^{2}
$$

and

$$
\langle D(\mathbf{u}, u), v\rangle:=d(\omega, u, v) \quad \forall \mathbf{u} \in \mathbf{H}, u, v \in V .
$$

These are all bounded operators in the corresponding spaces, and we have, due to the divergence-free condition on $\mathbf{u}$, that

$$
b_{T}(\mathbf{u}, T, T)=0, b_{q}(\mathbf{u}, q, q)=0
$$

for any $T, q \in V$.

The following estimate is easy.

Lemma 2.1 (boundedness of the functionals). Assume $U, U^{b} \in V^{2}$ and $\mathbf{u} \in \mathbf{V}$. There exist universal positive constants $\lambda$ and $K_{i}, 1 \leq i \leq 6$, such that

$\left|a_{T}\left(T, T^{b}\right)\right| \leq K_{1}\|T\|\left\|T^{b}\right\|, a_{T}(T, T) \geq \lambda\|T\|^{2} ;\left|a_{q}\left(q, q^{b}\right)\right| \leq K_{2}\|q\|\left\|q^{b}\right\|, a_{q}(q, q) \geq \lambda\|q\|^{2} ;$

$$
\left|l_{T}\left(T^{b}\right)\right| \leq K_{3}\left\|T^{b}\right\|,\left|l_{q}\left(q^{b}\right)\right| \leq K_{4}\left\|q^{b}\right\| ;\left|b\left(\mathbf{u}, U, U^{b}\right)\right| \leq K_{5}\|\mathbf{u}\|_{\mathbf{V}}|U|_{L^{2}}^{\frac{1}{2}}\|U\|^{\frac{1}{2}}\left\|U^{b}\right\| ;
$$

$$
\left|d\left(\omega, T, T^{b}\right)\right| \leq K_{6}|\omega|_{L^{2}}|T|_{L^{2}}^{\frac{1}{4}}\|T\|^{\frac{3}{4}}\left|T^{b}\right|_{L^{2}}^{\frac{1}{4}}\left\|T^{b}\right\|^{\frac{3}{4}} .
$$

2.3. Formulation of the problem and definition of solutions. Let $\left(T_{0}, q_{0}\right) \in$ $H \times H$ be such that $0 \leq q_{0} \leq 1$ a.e. in $\mathcal{M}$, and let $t_{1}>0$ be fixed. The weak formulation of problem (2.1)-(2.8) proposed in [31] is as follows:

Find a vector $U=(T, q) \in L^{2}\left(0, t_{1} ; V \times V\right) \cap C\left(\left[0, t_{1}\right] ; H \times H\right)$ with $\left(\partial_{t} T, \partial_{t} q\right) \in$ $L^{2}\left(0, t_{1} ; V^{*} \times V^{*}\right)$ such that, for almost every $t \in\left[0, t_{1}\right]$ and for every $\left(T^{b}, q^{b}\right) \in V \times \mathcal{K}$,

Copyright $@$ by SIAM. Unauthorized reproduction of this article is prohibited. 
we have

$\left\langle\partial_{t} T, T^{b}\right\rangle+a_{T}\left(T, T^{b}\right)+b_{T}\left(\mathbf{u}, T, T^{b}\right)-d\left(\omega, T, T^{b}\right)-l_{T}\left(T^{b}\right)=\left(\frac{1}{p} \omega^{-}(t) h_{q} \varphi(T), T^{b}\right)$,

$\left\langle\partial_{t} q, q^{b}-q\right\rangle+a_{q}\left(q, q^{b}-q\right)+b_{q}\left(\mathbf{u}, q, q^{b}-q\right)-l_{q}\left(q^{b}-q\right) \geq\left(-\frac{1}{p} \omega^{-}(t) h_{q} F(T), q^{b}-q\right)$

for some $h_{q} \in \mathcal{H}\left(q-q_{s}\right)$ and

$$
U_{0}=\left(T_{0}, q_{0}\right) .
$$

The existence of solutions to (2.23)-(2.25) was obtained in [31].

\section{Time discretization - the Euler scheme.}

3.1. Time discretization. We assume that the velocity field $\mathbf{u}$ is given, is timedependent, and satisfies $\mathbf{u} \in L^{r}\left(0, t_{1} ; V\right) \cap L^{\infty}\left(0, t_{1} ; H\right)$ for some given $r \in(4,+\infty]$.

Let $N$ be an integer which will later go to $+\infty$, and set $\Delta t:=k=t_{1} / N$. We will define recursively a family of elements of $V \times \mathcal{K}$, say $\left(T^{m}, q^{m}\right), m=0,1, \ldots, N$, where $\left(T^{m}, q^{m}\right)$ is intended to be an approximation of $(T, q)$ at time $m \Delta t$.

First, we define $\mathbf{u}^{m}, \omega^{m}$ for $m=1, \ldots, N$ :

$$
\mathbf{u}^{m}=\frac{1}{k} \int_{(m-1) k}^{m k} \mathbf{u}(t) d t, \quad \omega^{m}=\frac{1}{k} \int_{(m-1) k}^{m k} \omega d t .
$$

From the definition of $\mathbf{u}^{m}$, we observe that it inherits the divergence-free property of $\mathbf{u}$, and also

$$
\left|\mathbf{u}^{m}\right|_{L^{2}}=\left|\frac{1}{k} \int_{(m-1) k}^{m k} \mathbf{u}(t) d t\right|_{L^{2}} \leq \frac{1}{k} \int_{(m-1) k}^{m k}|\mathbf{u}(t)|_{L^{2}} d t \leq|\mathbf{u}|_{L^{\infty}\left(0, t_{1} ; H\right)} .
$$

We begin with $\left(T^{0}, q^{0}\right):=\left(T_{0}, q_{0}\right)$, i.e., the given initial datum, and when $\left(T^{0}, q^{0}\right)$, $\left(T^{1}, q^{1}\right), \ldots,\left(T^{m-1}, q^{m-1}\right)$ are known, $T^{m} \in V$ and $q^{m} \in \mathcal{K}$ are determined by

$$
\begin{array}{r}
\left\langle\frac{T^{m}-T^{m-1}}{k}, T^{b}\right\rangle+a_{T}\left(T^{m}, T^{b}\right)+b_{T}\left(\mathbf{u}^{m}, T^{m}, T^{b}\right)-d\left(\omega^{m}, T^{m-1}, T^{b}\right)-l_{T}\left(T^{b}\right) \\
=\left(\frac{1}{p}\left[\omega^{m}\right]^{-} h_{Q^{m}} \varphi\left(T^{m-1}\right), T^{b}\right),
\end{array}
$$

$$
\begin{array}{r}
\left\langle\frac{q^{m}-q^{m-1}}{k}, q^{b}-q^{m}\right\rangle+a_{q}\left(q^{m}, q^{b}-q^{m}\right)+b_{q}\left(\mathbf{u}^{m}, q^{m}, q^{b}-q^{m}\right)-l_{q}\left(q^{b}-q^{m}\right) \\
\geq\left(-\frac{1}{p}\left[\omega^{m}\right]^{-} h_{Q^{m}} F\left(T^{m-1}\right), q^{b}-q^{m}\right)
\end{array}
$$

for some $h_{Q^{m}} \in \mathcal{H}\left(Q^{m}-q_{s}\right)$, where $Q^{m}$ is either $q^{m-1}$ or $q^{m}$.

To prove the existence of a solution to (3.3)-(3.4), we proceed by approximation. Let $\varepsilon=\left(\varepsilon_{1}, \varepsilon_{2}\right)$ and $\varepsilon_{i}>0$ small for $i=1,2$. For $\varepsilon_{2}>0$, we define the regularization 
$H_{\varepsilon_{2}}$ of $\mathcal{H}(\cdot): \mathbb{R} \rightarrow[0,1]$ : equal to 0 for $r \geq 0$, equal to 1 for $r \geq \varepsilon_{2}$, and linear continuous between 0 and $\varepsilon_{2}$.

To show the existence of a solution $\left(T^{m}, q^{m}\right)$ to $(3.3)$, (3.4), we consider the following associated regularized and penalized problem:

Find $T_{\varepsilon}^{m}, q_{\varepsilon}^{m} \in V$ such that

$$
\begin{array}{r}
\left\langle\frac{T_{\varepsilon}^{m}-T_{\varepsilon}^{m-1}}{k}, T^{b}\right\rangle+a_{T}\left(T_{\varepsilon}^{m}, T^{b}\right)+b_{T}\left(\mathbf{u}^{m}, T_{\varepsilon}^{m}, T^{b}\right)-d\left(\omega^{m}, T_{\varepsilon}^{m-1}, T^{b}\right)-l_{T}\left(T^{b}\right) \\
=\left(\frac{1}{p}\left[\omega^{m}\right]^{-} H_{\varepsilon_{2}}\left(Q_{\varepsilon}^{m}-q_{s}\right) \varphi\left(T_{\varepsilon}^{m-1}\right), T^{b}\right)
\end{array}
$$

$$
\begin{array}{r}
\left\langle\frac{q_{\varepsilon}^{m}-q_{\varepsilon}^{m-1}}{k}, q^{b}\right\rangle+a_{q}\left(q_{\varepsilon}^{m}, q^{b}\right)+b_{q}\left(\mathbf{u}^{m}, q_{\varepsilon}^{m}, q^{b}\right)-l_{q}\left(q^{b}\right) \\
=\left(\frac{1}{\varepsilon_{1}}\left[q_{\varepsilon}^{m}\right]^{-}, q^{b}\right)-\left(\frac{1}{\varepsilon_{1}}\left[q_{\varepsilon}^{m}-1\right]^{+}, q^{b}\right)-\left(\frac{1}{p}\left[\omega^{m}\right]^{-} H_{\varepsilon_{2}}\left(Q_{\varepsilon}^{m}-q_{s}\right) F\left(T_{\varepsilon}^{m-1}\right), q^{b}\right)
\end{array}
$$

for all $T^{b}, q^{b} \in V$.

Remark 3.1. In the discretization, we have made the term $-d\left(\omega^{m}, T_{\varepsilon}^{m-1}, T^{b}\right)$ explicit by using $T_{\varepsilon}^{m-1}$ instead of $T_{\varepsilon}^{m}$. This will make the following steps of the proof simpler.

Remark 3.2. We will consider two choices for $Q_{\varepsilon}^{m}$ : either $Q_{\varepsilon}^{m}=q_{\varepsilon}^{m-1}$ or $Q_{\varepsilon}^{m}=$ $q_{\varepsilon}^{m}$. We call the corresponding schemes Scheme A and Scheme B.

3.2. Existence of $\left(\boldsymbol{T}_{\varepsilon}^{\boldsymbol{m}}, \boldsymbol{q}_{\varepsilon}^{\boldsymbol{m}}\right)$. Depending on the choices of $Q_{\varepsilon}^{m}$ in our scheme (3.5)-(3.6) as specified in Remark 3.2, we will consider the following two schemes: Scheme A and Scheme B.

Scheme A: $Q_{\varepsilon}^{m}=q_{\varepsilon}^{m-1}$. We notice that the factor $H_{\varepsilon_{2}}(\cdot)$ is known when we proceed to obtain $T_{\varepsilon}^{m}$ and $q_{\varepsilon}^{m}$ once $T_{\varepsilon}^{m-1}$ and $q_{\varepsilon}^{m-1}$ are achieved. We have the following lemma concerning the existence of the iteration sequence $\left(T_{\varepsilon}^{m}, q_{\varepsilon}^{m}\right)$ for $m=$ $1,2,3, \ldots$, given the initial datum.

LEMma 3.3. Let $\varepsilon_{1}, \varepsilon_{2}$ be arbitrary but fixed positive constants, $N$ be an arbitrary fixed integer, and $k=\frac{t_{1}}{N}, 1 \leq m \leq N$. Given $\left(T_{\varepsilon}^{m-1}, q_{\varepsilon}^{m-1}\right)$ and $Q_{\varepsilon}^{m}=q_{\varepsilon}^{m-1}$, the variational equalities $(3.5)-(3.6)$ on $\left(T_{\varepsilon}^{m}, q_{\varepsilon}^{m}\right)$ admit at least one solution.

Scheme B: $Q_{\varepsilon}^{m}=q_{\varepsilon}^{m}$. We note that unlike Scheme A, the factor $H_{\varepsilon_{2}}(\cdot)$ is not known when we proceed to obtain $T_{\varepsilon}^{m}$ and $q_{\varepsilon}^{m}$ even though $T_{\varepsilon}^{m-1}$ and $q_{\varepsilon}^{m-1}$ are known. We have the following lemma concerning the existence of the iteration sequence $\left(T_{\varepsilon}^{m}, q_{\varepsilon}^{m}\right)$ for $m=1,2,3, \ldots$, given the initial datum. Its proof is essentially the same as that of Theorem I-1.2 of [27] by the Galerkin method. See also [20, 26]. For Lemma 3.4 (and it would be similar for Lemma 3.3) we give only the main coercivity estimate used in the Galerkin construction.

LEMma 3.4. Let $\varepsilon_{1}, \varepsilon_{2}$ be arbitrary but fixed positive constants, $N$ be an arbitrary fixed integer, and $k=\frac{t_{1}}{N}, 1 \leq m \leq N$. Given $\left(T_{\varepsilon}^{m-1}, q_{\varepsilon}^{m-1}\right)$, the variational equalities (3.5)-(3.6) on $\left(T_{\varepsilon}^{m}, q_{\varepsilon}^{m}\right)$ with $Q_{\varepsilon}^{m}=q_{\varepsilon}^{m}$ admit at least one solution.

Sketch of proof of Lemma 3.4. Let $U=\left(T_{\varepsilon}^{m}, q_{\varepsilon}^{m}\right), \tilde{U}=\left(T_{\varepsilon}^{m-1}, q_{\varepsilon}^{m-1}\right)$, and $U^{b}=$ $\left(T^{b}, q^{b}\right)$. Then the variational inequalities (3.5)-(3.6) with $Q_{\varepsilon}^{m}=q_{\varepsilon}^{m}$ can be written 
as

$$
\begin{array}{r}
\left\langle\frac{U}{k}, U^{b}\right\rangle+a\left(U, U^{b}\right)+b\left(\mathbf{u}^{m}, U, U^{b}\right) \\
-\left(\frac{1}{\varepsilon_{1}} q^{-}, q^{b}\right)+\left(\frac{1}{\varepsilon_{1}}[q-1]^{+}, q^{b}\right)-d\left(\omega^{m}, \tilde{T}, T^{b}\right)-l\left(U^{b}\right) \\
-\left(\frac{1}{p}\left[\omega^{m}\right]^{-} H_{\varepsilon_{2}}\left(q-q_{s}\right) \varphi(\tilde{T}), T^{b}\right)+\left(\frac{1}{p}\left[\omega^{m}\right]^{-} H_{\varepsilon_{2}}\left(q-q_{s}\right) F(\tilde{T}), q^{b}\right)=\left\langle\frac{\tilde{U}}{k}, U^{b}\right\rangle .
\end{array}
$$

To proceed, we define $\left\langle P(U), U^{b}\right\rangle$ as

$$
\begin{array}{r}
\left\langle P(U), U^{b}\right\rangle=\left\langle\frac{U}{k}, U^{b}\right\rangle+a\left(U, U^{b}\right)+b\left(\mathbf{u}^{m}, U, U^{b}\right)-\left(\frac{1}{\varepsilon_{1}} q^{-}, q^{b}\right) \\
+\left(\frac{1}{\varepsilon_{1}}[q-1]^{+}, q^{b}\right)-d\left(\omega^{m}, T, T^{b}\right)-l\left(U^{b}\right)-\left(\frac{1}{p}\left[\omega^{m}\right]^{-} H_{\varepsilon_{2}}\left(q-q_{s}\right) \varphi(\tilde{T}), T^{b}\right) \\
+\left(\frac{1}{p}\left[\omega^{m}\right]^{-} H_{\varepsilon_{2}}\left(q-q_{s}\right) F(\tilde{T}), q^{b}\right)-\left\langle\frac{\tilde{U}}{k}, U^{b}\right\rangle .
\end{array}
$$

Therefore, we have

$$
\begin{array}{r}
\langle P(U), U\rangle=\left\langle\frac{U}{k}, U\right\rangle+a(U, U)+b\left(\mathbf{u}^{m}, U, U\right)-\left(\frac{1}{\varepsilon_{1}} q^{-}, q\right) \\
+\left(\frac{1}{\varepsilon_{1}}[q-1]^{+}, q\right)-d\left(\omega^{m}, \tilde{T}, T\right)-l(U)-\left(\frac{1}{p}\left[\omega^{m}\right]^{-} H_{\varepsilon_{2}}\left(q-q_{s}\right) \varphi(\tilde{T}), T\right) \\
+\left(\frac{1}{p}\left[\omega^{m}\right]^{-} H_{\varepsilon_{2}}\left(q-q_{s}\right) F(\tilde{T}), q\right)-\left\langle\frac{\tilde{U}}{k}, U\right\rangle .
\end{array}
$$

First, we observe that

$$
-\left(\frac{1}{\varepsilon_{1}} q^{-}, q\right)=-\left(\frac{1}{\varepsilon_{1}} q^{-}, q^{+}-q^{-}\right)=\frac{1}{\varepsilon_{1}}\left|q^{-}\right|_{L^{2}}^{2} \geq 0
$$

and

$$
\begin{aligned}
\left(\frac{1}{\varepsilon_{1}}[q-1]^{+}, q\right) & =\left(\frac{1}{\varepsilon_{1}}[q-1]^{+},[q-1]^{+}-[q-1]^{-}+1\right) \\
& =\frac{1}{\varepsilon_{1}}\left|[q-1]^{+}\right|_{L^{2}}^{2}+\left(\frac{1}{\varepsilon_{1}}[q-1]^{+}, 1\right) \geq 0 .
\end{aligned}
$$

Second, we notice that the term

$$
\begin{array}{r}
-d\left(\omega^{m}, \tilde{T}, T\right)-l(U)-\left(\frac{1}{p}\left[\omega^{m}\right]^{-} H_{\varepsilon_{2}}\left(q-q_{s}\right) \varphi(\tilde{T}), T\right) \\
+\left(\frac{1}{p}\left[\omega^{m}\right]^{-} H_{\varepsilon_{2}}\left(q-q_{s}\right) F(\tilde{T}), q\right)-\left\langle\frac{\tilde{U}}{k}, U\right\rangle
\end{array}
$$

is linear continuous in $U$ for $U \in V^{2}$. Therefore, its absolute value can be bounded by $\|U\|$ up to a constant depending on $\omega^{m}$ and $\tilde{T}$.

Finally, noticing that $b\left(\mathbf{u}^{m}, U, U\right)=0$, we have

$$
\langle P(U), U\rangle \geq \frac{1}{k}|U|_{L^{2}}^{2}+\lambda\|U\|^{2}-C\|U\|,
$$

which yields immediately the desired coercivity estimate.

Copyright $@$ by SIAM. Unauthorized reproduction of this article is prohibited. 
3.3. A priori estimates for $\left(T_{\varepsilon}^{m}, q_{\varepsilon}^{m}\right)$. We aim to obtain a priori estimates on $\left(T_{\varepsilon}^{m}, q_{\varepsilon}^{m}\right)$ independent of $k$ and $\varepsilon$ for the regularized and penalized problem (3.5)(3.6), which contains two schemes, Scheme A and Scheme B, according to different choices of $Q_{\varepsilon}^{m}$.

For notational convenience, we will suppress in this subsection the dependence on $\varepsilon$ in the expressions of $\left(T_{\varepsilon}^{m}, q_{\varepsilon}^{m}\right),\left(T_{\varepsilon}^{m-1}, q_{\varepsilon}^{m-1}\right)$, and $Q_{\varepsilon}^{m}$ in (3.5)-(3.6).

LEMMA 3.5. We have the estimates concerning the $U^{m}=\left(T^{m}, q^{m}\right)$ :

$$
\begin{aligned}
& \left|U^{j}\right|_{L^{2}}^{2} \leq C \quad \forall 1 \leq j \leq N ; \\
& \sum_{m=1}^{N}\left|U^{m}-U^{m-1}\right|_{L^{2}}^{2} \leq C, \quad k \sum_{m=1}^{N}\left\|U^{m}\right\|^{2} \leq C .
\end{aligned}
$$

Remark 3.6. Here and below $C$ denotes a finite constant depending on the data but independent of $\varepsilon$ and $k$; the constant $C$ may be different at different places.

To show Lemma 3.5, we begin with the following lemma.

Lemma 3.7. For each $m=1,2, \ldots, N, T^{m}$ and $q^{m}$ satisfy the following relations:

$$
\begin{gathered}
\left|T^{m}\right|_{L^{2}}^{2}-\left|T^{m-1}\right|_{L^{2}}^{2}+\left|T^{m}-T^{m-1}\right|_{L^{2}}^{2}+k \lambda\left\|T^{m}\right\|^{2} \leq C k\left(\left\|\omega^{m}\right\|^{2}\left|T^{m-1}\right|_{L^{2}}^{2}+1\right), \\
\quad\left|q^{m}\right|_{L^{2}}^{2}-\left|q^{m-1}\right|_{L^{2}}^{2}+\left|q^{m}-q^{m-1}\right|_{L^{2}}^{2}+k \lambda\left\|q^{m}\right\|^{2} \leq C k\left(\left|\omega^{m}\right|_{L^{2}}^{2}+1\right),
\end{gathered}
$$

where $C$ is a constant, independent of $\varepsilon$ and $k$.

Proof. Replacing $T^{b}$ by $2 k T^{m}$ in (3.5), we find

$$
\begin{array}{r}
2\left\langle T^{m}-T^{m-1}, T^{m}\right\rangle+2 k a_{T}\left(T^{m}, T^{m}\right)+2 k b_{T}\left(\mathbf{u}^{m}, T^{m}, T^{m}\right) \\
=2 k d\left(\omega^{m}, T^{m-1}, T^{m}\right)+2 k l_{T}\left(T^{m}\right)+2 k\left(\frac{1}{p}\left[\omega^{m}\right]^{-} H_{\varepsilon_{2}}\left(Q^{m}-q_{s}\right) \varphi\left(T^{m-1}\right), T^{m}\right) .
\end{array}
$$

By elementary calculation using (2.19)-(2.22), and arguments similar to those used in the proof of Lemma 3.4, we arrive at

$$
\left|T^{m}\right|_{L^{2}}^{2}-\left|T^{m-1}\right|_{L^{2}}^{2}+\left|T^{m}-T^{m-1}\right|_{L^{2}}^{2}+k \lambda\left\|T^{m}\right\|^{2} \leq C k\left(\left\|\omega^{m}\right\|^{2}\left|T^{m-1}\right|_{L^{2}}^{2}+1\right)
$$

Similarly, we infer from (3.6) that

$$
\begin{aligned}
2\left\langle q^{m}-q^{m-1}, q^{m}\right\rangle+ & 2 k a_{q}\left(q^{m}, q^{m}\right)+2 k b_{q}\left(\mathbf{u}^{m}, q^{m}, q^{m}\right)-2 k l_{q}\left(q^{m}\right) \\
& =2 k\left(\frac{1}{\varepsilon_{1}}\left[q^{m}\right]^{-}, q^{m}\right)-2 k\left(\frac{1}{\varepsilon_{1}}\left[q^{m}-1\right]^{+}, q^{m}\right) \\
& -2 k\left(\frac{1}{p}\left[\omega^{m}\right]^{-}(t) H_{\varepsilon_{2}}\left(Q^{m}-q_{s}\right) F\left(T^{m-1}\right), q^{m}\right) .
\end{aligned}
$$

By elementary calculation and using again (3.10), (3.11), we arrive at

$$
\left|q^{m}\right|_{L^{2}}^{2}-\left|q^{m-1}\right|_{L^{2}}^{2}+\left|q^{m}-q^{m-1}\right|_{L^{2}}^{2}+k \lambda\left\|q^{m}\right\|^{2} \leq C k\left(\left|\omega^{m}\right|_{L^{2}}^{2}+1\right) .
$$

The proof of Lemma 3.7 is complete.

Due to the term $\left\|\omega^{m}\right\|^{2}\left|T^{m-1}\right|_{L^{2}}^{2}$ appearing in the right-hand side of (3.13), we will need the following version of the discrete Gronwall lemma (see, e.g., [32]).

Copyright $@$ by SIAM. Unauthorized reproduction of this article is prohibited. 
Lemma 3.8 (discrete Gronwall lemma). Let $\theta$ be any positive constant and $N_{0}>1$ be an integer. Suppose the three nonnegative number sequences $\left(X_{m}\right),\left(Y_{m}\right)$, and $\left(Z_{m}\right)$ for $m=0,1,2, \ldots, N_{0}$ satisfy the following relation:

$$
X_{m} \leq X_{m-1}\left(1+\theta Y_{m}\right)+\theta Z_{m} .
$$

Then for $m=1,2,3, \ldots, N_{0}$, the following estimates hold:

$$
X_{m} \leq X_{0} \exp \left(\sum_{i=0}^{m-1} \theta Y_{i+1}\right)+\sum_{i=1}^{m-1} \theta Z_{i} \exp \left(\sum_{j=i}^{m-1} \theta Y_{j+1}\right)+\theta Z_{n}
$$

Proof of Lemma 3.5. If we identify $X_{m}=\left|T^{m}\right|_{L^{2}}^{2}, Y_{m}=C\left\|\omega^{m}\right\|^{2}, Z_{m}=C$, and $\theta=k,(3.13)$ is written as

$$
X_{m} \leq X_{m-1}\left(1+\theta Y_{m}\right)+\theta Z_{m}
$$

and

$$
\sum_{i=0}^{m-1} \theta Y_{i+1}=\sum_{i=0}^{m-1} k C\left\|\omega^{i+1}\right\|^{2} \leq C \sum_{i=0}^{N-1} k\left\|\omega^{i+1}\right\|^{2} \leq C|\omega|_{L^{2}\left(0, t_{1} ; V\right)}^{2},
$$

where the last inequality above follows from the triangle inequality and Hölder's inequality. By Lemma 3.8 and recalling that $N k=t_{1}$ and $k \leq t_{1}$, we find for $m=1,2, \ldots, N$,

$$
\left|T^{m}\right|_{L^{2}}^{2} \leq\left|T_{0}\right|_{L^{2}}^{2} \exp \left(C|\omega|_{L^{2}\left(0, t_{1} ; V\right)}^{2}\right)+C N k \exp \left(C|\omega|_{L^{2}\left(0, t_{1} ; V\right)}^{2}\right)+C k \leq C\left(U_{0}, \omega, t_{1}\right) .
$$

With the above bound, we obtain from (3.13) that

$$
\left|T^{m}\right|_{L^{2}}^{2}-\left|T^{m-1}\right|_{L^{2}}^{2}+\left|T^{m}-T^{m-1}\right|_{L^{2}}^{2}+k \lambda\left\|T^{m}\right\|^{2} \leq C\left(U_{0}, \omega, t_{1}\right) k\left\|\omega^{m}\right\|^{2}+C k .
$$

Summing these inequalities in $m$ from 1 to $N$, we obtain

$$
\left|T^{N}\right|_{L^{2}}^{2}-\left|T_{0}\right|_{L^{2}}^{2}+\sum_{m=1}^{N}\left|T^{m}-T^{m-1}\right|_{L^{2}}^{2}+k \lambda \sum_{m=1}^{N}\left\|T^{m}\right\|^{2} \leq C\left(U_{0}, \omega, t_{1}\right) \sum_{m=1}^{N} k\left\|\omega^{m}\right\|^{2}+C N k,
$$

which implies

$$
\left|T^{N}\right|_{L^{2}}^{2}+\sum_{m=1}^{N}\left|T^{m}-T^{m-1}\right|_{L^{2}}^{2}+k \lambda \sum_{m=1}^{N}\left\|T^{m}\right\|^{2} \leq C\left(U_{0}, \omega, t_{1}\right) \sum_{m=1}^{N} k\left\|\omega^{m}\right\|^{2}+C N k+\left|T_{0}\right|_{L^{2}}^{2} .
$$

Again, the right-hand side can be bounded by $C\left(U_{0}, \omega, t_{1}\right)$ since $\sum_{m=1}^{N} k\left\|\omega^{m}\right\|^{2} \lesssim$ $|\omega|_{L^{2}\left(0, t_{1} ; V\right)}^{2}$ as in (3.2).

The estimates for $q^{m}$ are more direct. Summing the inequalities (3.14) in $m$ from 1 to $j$ for any $j \leq N$ and dropping some positive terms, we obtain

$$
\left|q^{j}\right|_{L^{2}}^{2}-\left|q_{0}\right|_{L^{2}}^{2} \leq \sum_{m=1}^{j} C k\left(\left|\omega^{m}\right|_{L^{2}}^{2}+1\right) \leq C\left(q_{0}, \omega, t_{1}\right) .
$$

Therefore,

$$
\left|q^{j}\right|_{L^{2}}^{2} \leq\left|q_{0}\right|_{L^{2}}^{2}+C\left(q_{0}, \omega, t_{1}\right) \leq C\left(q_{0}, \omega, t_{1}\right)
$$

Copyright $@$ by SIAM. Unauthorized reproduction of this article is prohibited. 
Now, we sum the inequalities (3.14) in $m$ from 1 to $N$, and we obtain

$$
\left|q^{N}\right|_{L^{2}}^{2}-\left|q_{0}\right|_{L^{2}}^{2}+\sum_{m=1}^{N}\left|q^{m}-q^{m-1}\right|_{L^{2}}^{2}+k \sum_{m=1}^{N} \lambda\left\|q^{m}\right\|^{2} \leq C \sum_{m=1}^{N} k\left(\left|\omega^{m}\right|_{L^{2}}^{2}+1\right),
$$

which implies

$$
\sum_{m=1}^{N}\left|q^{m}-q^{m-1}\right|_{L^{2}}^{2}+k \sum_{m=1}^{N} \lambda\left\|q^{m}\right\|^{2} \leq C\left(q_{0}, \omega, t_{1}\right)
$$

The proof of Lemma 3.5 is complete.

Next, we will seek an a priori bound for the norm $k \sum_{m=1}^{N}\left\|\frac{U^{m}-U^{m-1}}{k}\right\|_{V^{*}}^{2}$, which will be used later in the compactness argument. We have the following lemma.

Lemma 3.9. For any $\varepsilon_{1}>0$ and any $\varepsilon_{2}>0$, the inequality

$$
k \sum_{m=1}^{N}\left\|\frac{U^{m}-U^{m-1}}{k}\right\|_{V^{*}}^{2} \leq C\left(\mathbf{u}, U_{0}, t_{1}\right)<+\infty
$$

holds for some constant $C\left(\mathbf{u}, U_{0}, t_{1}\right)$ depending on $U_{0}, \mathbf{u}, t_{1}$, but not on $\varepsilon$ and $k$.

The main point of Lemma 3.9 is that the bound is independent of $\varepsilon=\left(\varepsilon_{1}, \varepsilon_{2}\right)$ and $k$. As $\varepsilon_{2}$ comes into play through the regularization function $H_{\varepsilon_{2}}$ and $H_{\varepsilon_{2}}$ is bounded by 1 , it is easy to obtain the bound independent of $\varepsilon_{2}$. Therefore, the main issue here is to control the penalization terms which contain a blowup factor $\frac{1}{\varepsilon_{1}}$ in the limit process $\varepsilon \rightarrow(0+, 0+)$. This is done in Lemmas 3.10 and 3.11 .

LEMMA 3.10. The following bound holds:

$$
k \sum_{m=1}^{N}\left|\frac{\left[q^{m}\right]^{-}}{\varepsilon_{1}}\right|_{L^{2}}^{2} \leq C|\omega|_{L^{2}\left(0, t_{1} ; H\right)}^{2} .
$$

Proof. We set $q^{b}=\left[q^{m}\right]^{-} \in V$ in (3.6) and find

$$
\begin{aligned}
\left\langle\frac{q^{m}-q^{m-1}}{k},\left[q^{m}\right]^{-}\right\rangle+ & a_{q}\left(q^{m},\left[q^{m}\right]^{-}\right)+b_{q}\left(\mathbf{u}^{m}, q^{m},\left[q^{m}\right]^{-}\right)-l_{q}\left(\left[q^{m}\right]^{-}\right) \\
= & \left(\frac{1}{\varepsilon_{1}}\left[q^{m}\right]^{-},\left[q^{m}\right]^{-}\right)-\left(\frac{1}{\varepsilon_{1}}\left[q^{m}-1\right]^{+},\left[q^{m}\right]^{-}\right) \\
& -\left(\frac{1}{p}\left[\omega^{m}\right]^{-} H_{\varepsilon_{2}}\left(Q^{m}-q_{s}\right) F\left(T^{m-1}\right),\left[q^{m}\right]^{-}\right) .
\end{aligned}
$$

When $q^{m} \geq 0$, notice that $-\left(\frac{1}{\varepsilon_{1}}\left[q^{m}-1\right]^{+},\left[q^{m}\right]^{-}\right)=0$.

By linearity and (2.19), we have

$$
\begin{aligned}
b_{q}\left(\mathbf{u}^{m}, q^{m},\left[q^{m}\right]^{-}\right) & =b_{q}\left(\mathbf{u}^{m},\left[q^{m}\right]^{+}-\left[q^{m}\right]^{-},\left[q^{m}\right]^{-}\right) \\
& =b_{q}\left(\mathbf{u}^{m},\left[q^{m}\right]^{+},\left[q^{m}\right]^{-}\right)-b_{q}\left(\mathbf{u}^{m},\left[q^{m}\right]^{-},\left[q^{m}\right]^{-}\right)=0 .
\end{aligned}
$$

By linearity and coercivity, we have

$$
a_{q}\left(q^{m},\left[q^{m}\right]^{-}\right)=a_{q}\left(\left[q^{m}\right]^{+},\left[q^{m}\right]^{-}\right)-a_{q}\left(\left[q^{m}\right]^{-},\left[q^{m}\right]^{-}\right) \leq 0 .
$$

Copyright (c) by SIAM. Unauthorized reproduction of this article is prohibited. 
In (3.27) and (3.28), we have used the fact that

$$
b_{q}\left(\mathbf{u}^{m},\left[q^{m}\right]^{+},\left[q^{m}\right]^{-}\right)=0, \quad a_{q}\left(\left[q^{m}\right]^{+},\left[q^{m}\right]^{-}\right)=0,
$$

because $\left[q^{m}\right]^{+}$vanishes on the support of $\left[q^{m}\right]^{-}$.

By the specific form of the functional $l_{q}$, we have $l_{q}\left(\left[q^{m}\right]^{-}\right) \geq 0$, and by Hölder's inequality, we have

$$
\left|-\left(\frac{1}{p}\left[\omega^{m}\right]^{-} H_{\varepsilon_{2}}\left(Q^{m}-q_{s}\right) F\left(T^{m-1}\right),\left[q^{m}\right]^{-}\right)\right| \leq C\left|\omega^{m}\right|_{L^{2}}\left|\left[q^{m}\right]^{-}\right|_{L^{2}} .
$$

In view of the above relations, we infer from (3.26) that

$$
\frac{1}{\varepsilon_{1}}\left|\left[q^{m}\right]^{-}\right|_{L^{2}}^{2} \leq\left\langle\frac{q^{m}-q^{m-1}}{k},\left[q^{m}\right]^{-}\right\rangle+C\left|\omega^{m}\right|_{L^{2}}\left|\left[q^{m}\right]^{-}\right|_{L^{2}} .
$$

By direct calculation, writing $q^{m}=\left[q^{m}\right]^{+}-\left[q^{m}\right]^{-}$and the same for $q^{m-1}$, we see that

$$
\sum_{m=1}^{N}\left\langle q^{m}-q^{m-1},\left[q^{m}\right]^{-}\right\rangle=-\frac{1}{2}\left|\left[q^{N}\right]^{-}\right|_{L^{2}}^{2} \leq 0 .
$$

Then we write

$$
C\left|\omega^{m}\right|_{L^{2}}\left|\left[q^{m}\right]^{-}\right|_{L^{2}} \leq \frac{1}{2 \varepsilon_{1}}\left|\left[q^{m}\right]^{-}\right|_{L^{2}}^{2}+C \varepsilon_{1}\left|\omega^{m}\right|_{L^{2}}^{2} .
$$

From (3.30) and (3.32), we have

$$
\frac{1}{2 \varepsilon_{1}}\left|\left[q^{m}\right]^{-}\right|_{L^{2}}^{2} \leq\left\langle\frac{q^{m}-q^{m-1}}{k},\left[q^{m}\right]^{-}\right\rangle+C \varepsilon_{1}\left|\omega^{m}\right|_{L^{2}}^{2} .
$$

Summing in $m$ from 1 to $N$ and in view of (3.31), we infer from (3.33) that

$$
\sum_{m=1}^{N} \frac{1}{2 \varepsilon_{1}^{2}}\left|\left[q^{m}\right]^{-}\right|_{L^{2}}^{2} \leq C \sum_{m=1}^{N}\left|\omega^{m}\right|_{L^{2}}^{2}
$$

Multiplying (3.34) by $2 k$, we obtain

$$
k \sum_{m=1}^{N} \frac{1}{\varepsilon_{1}^{2}}\left|\left[q^{m}\right]^{-}\right|_{L^{2}}^{2} \leq C k \sum_{m=1}^{N}\left|\omega^{m}\right|_{L^{2}}^{2} \leq C|\omega|_{L^{2}\left(0, t_{1} ; H\right)}^{2} .
$$

The proof of Lemma 3.10 is complete.

LEMMA 3.11. The following bound holds:

$$
k \sum_{m=1}^{N}\left|\frac{\left[q^{m}-1\right]^{+}}{\varepsilon_{1}}\right|_{L^{2}}^{2} \leq C|\omega|_{L^{2}\left(0, t_{1} ; H\right)}^{2} .
$$

The proof of Lemma 3.11 is very similar to that of Lemma 3.10. We skip the details.

We can now turn to the proof of Lemma 3.9.

Copyright $@$ ( ) by SIAM. Unauthorized reproduction of this article is prohibited. 
Proof of Lemma 3.9. We estimate the duality pair $\left\langle\frac{U^{m}-U^{m-1}}{k}, U^{b}\right\rangle$, where $U^{b}=$ $\left(T^{b}, q^{b}\right) \in V^{2}$. By adding (3.5) and (3.6), we can write

$$
\begin{array}{r}
\left|\left\langle\frac{U^{m}-U^{m-1}}{k}, U^{b}\right\rangle\right|=\mid-a\left(U^{m}, U^{b}\right)-b\left(\mathbf{u}^{m}, U^{m}, U^{b}\right) \\
+\left(\frac{1}{\varepsilon_{1}}\left[q^{m}\right]^{-}, q^{b}\right)-\left(\frac{1}{\varepsilon_{1}}\left[q^{m}-1\right]^{+}, q^{b}\right)+d\left(\omega^{m}, T^{m-1}, T^{b}\right)+l\left(U^{b}\right) \\
+\left(\frac{1}{p}\left[\omega^{m}\right]^{-} H_{\varepsilon_{2}}\left(Q^{m}-q_{s}\right) \varphi\left(T^{m-1}\right), T^{b}\right)-\left(\frac{1}{p}\left[\omega^{m}\right]^{-} H_{\varepsilon_{2}}\left(Q^{m}-q_{s}\right) F\left(T^{m-1}\right), q^{b}\right) \mid .
\end{array}
$$

The right-hand side of (3.36) is bounded using (2.20)-(2.22) and the similar arguments used in Lemma 3.10; then we obtain

$$
\begin{aligned}
\left\|\frac{U^{m}-U^{m-1}}{k}\right\|_{V^{*}} \leq & C\left(\left\|U^{m}\right\|+\left\|T^{m-1}\right\|\right)+C\left\|\mathbf{u}^{m}\right\|\left\|U^{m}\right\|^{1 / 2} \\
& +\frac{1}{\varepsilon_{1}}\left|\left[q^{m}\right]^{-}\right|_{L^{2}}+\frac{1}{\varepsilon_{1}}\left|\left[q^{m}-1\right]^{+}\right|_{L^{2}}+C,
\end{aligned}
$$

where the absolute constants $C$ may depend on $\mathbf{u}, U_{0}, t_{1}$, but not on $\varepsilon$ and $k$.

Writing $\left\|\mathbf{u}^{m}\right\|^{2}\left\|U^{m}\right\| \leq\left\|\mathbf{u}^{m}\right\|^{4}+\left\|U^{m}\right\|^{2}$, we infer from (3.37) that

$$
\begin{aligned}
& k \sum_{m=1}^{N}\left\|\frac{U^{m}-U^{m-1}}{k}\right\|_{V^{*}}^{2} \\
\leq & C \sum_{m=1}^{N} k\left(\left\|U^{m}\right\|^{2}+\left\|\mathbf{u}^{m}\right\|^{4}+1\right)+k \sum_{m=1}^{N}\left|\frac{\left[q^{m}\right]^{-}}{\varepsilon_{1}}\right|_{L^{2}}^{2}+k \sum_{m=1}^{N}\left|\frac{\left[q^{m}-1\right]^{+}}{\varepsilon_{1}}\right|_{L^{2}}^{2} .
\end{aligned}
$$

For the term $\sum_{m=1}^{N} k\left\|\mathbf{u}^{m}\right\|^{4}$ in the right-hand side of (3.38), we could control it by the triangle inequality and Hölder's inequality as follows:

$$
\begin{aligned}
\sum_{m=1}^{N} k\left\|\mathbf{u}^{m}\right\|^{4} & =\sum_{m=1}^{N} k\left\|\frac{1}{k} \int_{(m-1) k}^{m k} \mathbf{u}(t) d t\right\|^{4} \leq \sum_{m=1}^{N} k\left(\frac{1}{k} \int_{(m-1) k}^{m k}\|u(t)\| d t\right)^{4} \\
& \leq \sum_{m=1}^{N} k\left(\frac{1}{k} \int_{(m-1) k}^{m k}\|u(t)\|^{4} d t\right)=\sum_{m=1}^{N} \int_{(m-1) k}^{m k}\|u(t)\|^{4} d t=|\mathbf{u}|_{L^{4}\left(0, t_{1} ; V\right)}^{4}
\end{aligned}
$$

All terms in the right-hand side of (3.38) are bounded by a constant $C$ independent of $\varepsilon$ and $k$, thanks to the previous estimates and, in particular, the estimates in Lemmas 3.10 and 3.11. This shows that

$$
k \sum_{m=1}^{N}\left\|\frac{U^{m}-U^{m-1}}{k}\right\|_{V^{*}}^{2} \leq C\left(\mathbf{u}, U_{0}, t_{1}\right)<+\infty .
$$

The proof of Lemma 3.9 is complete.

3.4. Passage to the limit $\varepsilon \rightarrow(0+, 0+)$. In this subsection, we will make explicit the dependence of $U_{\varepsilon}^{m}$ on $\varepsilon$. Let $k>0$ be fixed. Our goal is to pass to the limit $\varepsilon \rightarrow(0+, 0+)$ in the scheme (3.5)-(3.6) with the aim of obtaining the existence 
of solutions to the time discretized scheme (3.3)-(3.4). Though the convergences in our arguments below may be up to subsequences, we will not always specify this fact and we also will not elaborate on the indices.

After extracting a finite number of subsequences, $\varepsilon \rightarrow 0$, we infer from Lemma 3.5 that for $m=1,2, \ldots, N$, there exist functions $U^{m} \in V$ such that as $\varepsilon \rightarrow 0+$,

$$
U_{\varepsilon}^{m} \rightarrow U^{m} \text { weakly in } V \text { and strongly in } H,
$$

since the inclusion $V \subset H$ is compact. We still use $\varepsilon$ as the index for the subsequence.

By an additional extraction of subsequences,

$$
U_{\varepsilon}^{m}(x) \rightarrow U^{m}(x) \text { a.e., } \quad m=1,2, \ldots, N .
$$

Meanwhile, we have

$$
H_{\varepsilon_{2}}\left(Q_{\varepsilon}^{m}-q_{s}\right) \rightarrow h_{Q^{m}} \text { weak-* in } L^{\infty}(\mathcal{M}) \text { for } m=1,2, \ldots, N .
$$

Concerning the limit function $q^{m}$, the second component of $U^{m}$ for $m=1,2, \ldots, N$, we know from Lemmas 3.10 and 3.11 that

$$
k \sum_{m=1}^{N}\left(\left|\left[q_{\varepsilon}^{m}\right]^{-}\right|_{L^{2}}^{2}+\left|\left[q_{\varepsilon}^{m}-1\right]^{+}\right|_{L^{2}}^{2}\right) \leq C \varepsilon_{1}^{2}|\omega|_{L^{2}\left(0, t_{1} ; H\right)}^{2}
$$

As the real functions $g_{ \pm}(\theta)=\theta^{ \pm}$are both Lipschitz functions with Lipschitz constant 1 on $\mathbb{R}$, we have

$$
\left|\left[q_{\varepsilon}^{m}\right]^{-}-\left[q^{m}\right]^{-}\right|_{L^{2}} \leq\left|q_{\varepsilon}^{m}-q^{m}\right|_{L^{2}}, \quad\left|\left[q_{\varepsilon}^{m}-1\right]^{+}-\left[q^{m}-1\right]^{+}\right|_{L^{2}} \leq\left|q_{\varepsilon}^{m}-q^{m}\right|_{L^{2}} .
$$

Consequently, with (3.40) we have $\left[q_{\varepsilon}^{m}\right]^{-} \rightarrow\left[q^{m}\right]^{-}$and $\left[q_{\varepsilon}^{m}-1\right]^{+} \rightarrow\left[q^{m}-1\right]^{+}$in $H$. As $k>0$ is a fixed number, we can pass to the limit on $\varepsilon$ in (3.42) to obtain that

$$
\sum_{m=1}^{N}\left(\left|\left[q^{m}\right]^{-}\right|_{L^{2}}^{2}+\left|\left[q^{m}-1\right]^{+}\right|_{L^{2}}^{2}\right)=0,
$$

which implies

$$
0 \leq q^{m} \leq 1 \text { a.e. in } \mathcal{M} \text {, i.e., } q^{m} \in \mathcal{K} \text {. }
$$

We now want to pass to the limit in (3.5) and (3.6). We first aim to derive the variational inequality (3.4) by passing to the limit in (3.6).

We replace $q^{b}$ in (3.6) by $q^{b}-q_{\varepsilon}^{m}$ with $q^{b} \in \mathcal{K}$ and arrive at the following equation:

$$
\begin{array}{r}
\left\langle\frac{q_{\varepsilon}^{m}-q_{\varepsilon}^{m-1}}{k}, q^{b}-q_{\varepsilon}^{m}\right\rangle+a_{q}\left(q_{\varepsilon}^{m}, q^{b}-q_{\varepsilon}^{m}\right)+b_{q}\left(\mathbf{u}^{m}, q_{\varepsilon}^{m}, q^{b}-q_{\varepsilon}^{m}\right)-l_{q}\left(q^{b}-q_{\varepsilon}^{m}\right) \\
=\left(\frac{1}{\varepsilon_{1}}\left[q_{\varepsilon}^{m}\right]^{-}, q^{b}-q_{\varepsilon}^{m}\right)-\left(\frac{1}{\varepsilon_{1}}\left[q_{\varepsilon}^{m}-1\right]^{+}, q^{b}-q_{\varepsilon}^{m}\right) \\
-\left(\frac{1}{p}\left[\omega^{m}\right]^{-} H_{\varepsilon_{2}}\left(Q_{\varepsilon}^{m}-q_{s}\right) F\left(T_{\varepsilon}^{m-1}\right), q^{b}-q_{\varepsilon}^{m}\right) .
\end{array}
$$

Copyright (c) by SIAM. Unauthorized reproduction of this article is prohibited. 
Concerning the penalization terms, we have, as before, the following two inequalities:

$$
\begin{aligned}
\left(\frac{1}{\varepsilon_{1}}\left[q_{\varepsilon}^{m}\right]^{-}, q^{b}-q_{\varepsilon}^{m}\right) & =\left(\frac{1}{\varepsilon_{1}}\left[q_{\varepsilon}^{m}\right]^{-}, q^{b}-\left[q_{\varepsilon}^{m}\right]^{+}+\left[q_{\varepsilon}^{m}\right]^{-}\right) \\
& =\left(\frac{1}{\varepsilon_{1}}\left[q_{\varepsilon}^{m}\right]^{-}, q^{b}\right)+\frac{1}{\varepsilon_{1}}\left|\left[q_{\varepsilon}^{m}\right]^{-}\right|_{L^{2}}^{2} \geq 0,
\end{aligned}
$$

$$
\begin{aligned}
-\left(\frac{1}{\varepsilon_{1}}\left[q_{\varepsilon}^{m}-1\right]^{+}, q^{b}-q_{\varepsilon}^{m}\right) & =-\left(\frac{1}{\varepsilon_{1}}\left[q_{\varepsilon}^{m}-1\right]^{+},-\left(1-q^{b}\right)-\left[q_{\varepsilon}^{m}-1\right]^{+}+\left[q_{\varepsilon}^{m}-1\right]^{-}\right) \\
& =\left(\frac{1}{\varepsilon_{1}}\left[q_{\varepsilon}^{m}-1\right]^{+},\left(1-q^{b}\right)\right)+\frac{1}{\varepsilon_{1}}\left|\left[q_{\varepsilon}^{m}-1\right]^{+}\right|_{L^{2}}^{2} \geq 0,
\end{aligned}
$$

where we have used the fact that $0 \leq q^{b} \leq 1$ since $q^{b} \in \mathcal{K}$.

As $q^{b}-q_{\varepsilon}^{m} \rightarrow q^{b}-q^{m}$ weakly in $V$, we also have $l_{q}\left(q^{b}-q_{\varepsilon}^{m}\right) \rightarrow l_{q}\left(q^{b}-q^{m}\right)$.

To pass to the limit in $a_{q}\left(q_{\varepsilon}^{m}, q^{b}-q_{\varepsilon}^{m}\right)$, we use the weak convergence of $q^{m}$ in $V$ and the weak lower semicontinuity property of the norm. Hence,

$$
\begin{aligned}
\limsup a_{q}\left(q_{\varepsilon}^{m}, q^{b}-q_{\varepsilon}^{m}\right) & =\lim a_{q}\left(q_{\varepsilon}^{m}, q^{b}\right)-\liminf a_{q}\left(q_{\varepsilon}^{m}, q_{\varepsilon}^{m}\right) \\
& \leq a_{q}\left(q^{m}, q^{b}\right)-a_{q}\left(q^{m}, q^{m}\right)=a_{q}\left(q^{m}, q^{b}-q^{m}\right) .
\end{aligned}
$$

For the term $b_{q}\left(\mathbf{u}^{m}, q_{\varepsilon}^{m}, q^{b}-q_{\varepsilon}^{m}\right)$, since $\operatorname{div} u^{m}=0$, equation (2.19) implies that $b_{q}\left(\mathbf{u}^{m}, q_{\varepsilon}^{m}, q_{\varepsilon}^{m}\right)=0$ and $b_{q}\left(\mathbf{u}^{m}, q^{m}, q^{m}\right)=0$. Then by applying (2.21), we write

$$
\begin{aligned}
\left|b_{q}\left(\mathbf{u}^{m}, q_{\varepsilon}^{m}, q^{b}-q_{\varepsilon}^{m}\right)-b_{q}\left(\mathbf{u}^{m}, q^{m}, q^{b}-q^{m}\right)\right| & =\left|b_{q}\left(\mathbf{u}^{m}, q_{\varepsilon}^{m}-q^{m}, q^{b}\right)\right| \\
& \leq C\left\|\mathbf{u}^{m}\right\| \mathbf{v}\left|q_{\varepsilon}^{m}-q^{m}\right|_{L^{2}}^{\frac{1}{2}}\left\|q_{\varepsilon}^{m}-q^{m}\right\|^{\frac{1}{2}}\left\|q^{b}\right\| .
\end{aligned}
$$

Note that $\mathbf{u}^{m}$ and $q^{b}$ do not depend on $\varepsilon$. Due to the strong convergence of $q_{\varepsilon}^{m}$ to $q^{m}$ in $H$ and the boundedness of $\left\|q_{\varepsilon}^{m}-q^{m}\right\|$, we conclude that

$$
b_{q}\left(\mathbf{u}^{m}, q_{\varepsilon}^{m}, q^{b}-q_{\varepsilon}^{m}\right) \rightarrow b_{q}\left(\mathbf{u}^{m}, q^{m}, q^{b}-q^{m}\right) .
$$

Now, we consider the term $-\left(\frac{1}{p}\left[\omega^{m}\right]^{-} H_{\varepsilon_{2}}\left(Q_{\varepsilon}^{m}-q_{s}\right) F\left(T_{\varepsilon}^{m-1}\right), q^{b}-q_{\varepsilon}^{m}\right)$. In view of the convergences $H_{\varepsilon_{2}}\left(Q_{\varepsilon}^{m}-q_{s}\right) \rightarrow h_{Q^{m}}$ weak-* in $L^{\infty}(\mathcal{M}), U_{\varepsilon}^{m} \rightarrow U^{m}$ strongly in $H$, and noticing the fact that the function $F$ is a globally Lipschitz function, we see, by an additional extraction of subsequences, with (3.41) that $F\left(U_{\varepsilon}^{m}(x)\right) \rightarrow F\left(U^{m}(x)\right)$ a.e. Then, by the Lebesgue dominated convergence theorem, $F\left(U_{\varepsilon}^{m}\right) \rightarrow F\left(U^{m}\right)$ strongly in $H$, and therefore $H_{\varepsilon_{2}}\left(Q_{\varepsilon}^{m}-q_{s}\right) F\left(T_{\varepsilon}^{m-1}\right) \rightarrow h_{Q^{m}} F\left(T^{m-1}\right)$ weakly in $H$. Together with the strong convergence of $q^{b}-q_{\varepsilon}^{m}$ to $q^{b}-q^{m}$ in $H$, we obtain

$$
-\left(\frac{1}{p}\left[\omega^{m}\right]^{-} H_{\varepsilon_{2}}\left(Q_{\varepsilon}^{m}-q_{s}\right) F\left(T_{\varepsilon}^{m-1}\right), q^{b}-q_{\varepsilon}^{m}\right) \rightarrow-\left(\frac{1}{p}\left[\omega^{m}\right]^{-} h_{Q^{m}} F\left(T^{m-1}\right), q^{b}-q^{m}\right) .
$$

Due to the strong convergence $U_{\varepsilon}^{m} \rightarrow U^{m}$ in $H$ and the continuity of the $L^{2}$ inner product, we have

$$
\left\langle\frac{q_{\varepsilon}^{m}-q_{\varepsilon}^{m-1}}{k}, q^{b}-q_{\varepsilon}^{m}\right\rangle=\int_{\mathcal{M}} \frac{q_{\varepsilon}^{m}-q_{\varepsilon}^{m-1}}{k}\left(q^{b}-q_{\varepsilon}^{m}\right) d \mathcal{M} \rightarrow\left\langle\frac{q^{m}-q^{m-1}}{k}, q^{b}-q^{m}\right\rangle .
$$

In view of (3.45)-(3.50), together with (3.43), we conclude, after passing to the limit on $\varepsilon$ in (3.44), that (3.4) is satisfied.

Copyright $@$ by SIAM. Unauthorized reproduction of this article is prohibited. 
Second, we derive the variational equality (3.3) from (3.5) by passing to the limit on $\varepsilon$. The proof is similar to and easier than the one for $q_{\varepsilon}^{m}$, and we skip the details.

Summarizing the above arguments, we obtain from (3.5) through passing to the limit on $\varepsilon$ that (3.3) holds true.

It remains to show that $h_{Q^{m}} \in \mathcal{H}\left(Q^{m}-q_{s}\right)$. For that purpose, we consider the following antiderivative $K_{\varepsilon_{2}}$ of the function $H_{\varepsilon_{2}}$ :

$$
K_{\varepsilon_{2}}(r)=0 \text { if } r \leq 0 ;=\frac{r^{2}}{2 \varepsilon_{2}} \text { if } r \in\left(0, \varepsilon_{2}\right] ;=r-\frac{\varepsilon_{2}}{2} \text { if } r>\varepsilon_{2} .
$$

We easily see that both $H_{\varepsilon_{2}}$ and $K_{\varepsilon_{2}}$ are Lipschitz functions and that the following inequalities hold for any $r_{1}, r_{2} \in \mathbb{R}$ :

$$
\left|H_{\varepsilon_{2}}\left(r_{1}\right)-H_{\varepsilon_{2}}\left(r_{2}\right)\right| \leq \frac{1}{\varepsilon_{2}}\left|r_{1}-r_{2}\right|, \quad\left|K_{\varepsilon_{2}}\left(r_{1}\right)-K_{\varepsilon_{2}}\left(r_{2}\right)\right| \leq\left|r_{1}-r_{2}\right| .
$$

By the expression of $K_{\varepsilon_{2}}$, we observe that

$$
\left|K_{\varepsilon_{2}}(r)-r\right| \leq \frac{\varepsilon_{2}}{2} \quad \text { for } r \geq 0 .
$$

Consider now the functional $q \rightarrow\left(K_{\varepsilon_{2}}(q), 1\right)$ from $V$ to $R$. As the function $K_{\varepsilon_{2}}$ is a convex function on $\mathbb{R}$, we know that the functional $q \rightarrow\left(K_{\varepsilon_{2}}(q), 1\right)$ is convex on $V$. As the function $K_{\varepsilon_{2}}$ is continuously differentiable, the functional $q \rightarrow\left(K_{\varepsilon_{2}}(q), 1\right)$ is actually Fréchet differentiable with Fréchet derivative at $q$ equal to $H_{\varepsilon_{2}}(q) \in V^{*}$. In particular, by considering its Gâteaux derivative at the point $Q_{\varepsilon}^{m}-q_{s}$ along the direction $q^{b}-Q_{\varepsilon}^{m}$, we have the following inequality:

$$
\begin{aligned}
& \left(K_{\varepsilon_{2}}\left(q^{b}-q_{s}\right), 1\right)-\left(K_{\varepsilon_{2}}\left(Q_{\varepsilon}^{m}-q_{s}\right), 1\right) \\
= & \left(K_{\varepsilon_{2}}\left(Q_{\varepsilon}^{m}-q_{s}+q^{b}-Q_{\varepsilon}^{m}\right), 1\right)-\left(K_{\varepsilon_{2}}\left(Q_{\varepsilon}^{m}-q_{s}\right), 1\right) \geq\left\langle H_{\varepsilon_{2}}\left(Q_{\varepsilon}^{m}-q_{s}\right), q^{b}-Q_{\varepsilon}^{m}\right\rangle .
\end{aligned}
$$

As the duality pair $\left\langle H_{\varepsilon_{2}}\left(Q_{\varepsilon}^{m}-q_{s}\right), q^{b}-Q_{\varepsilon}^{m}\right\rangle$ can be realized by an $L^{2}$ inner product, and in view of the two convergences $H_{\varepsilon_{2}}\left(Q^{m}-q_{s}\right) \rightarrow h_{Q^{m}}$ weakly-* in $L^{\infty}(\mathcal{M})$ and $Q_{\varepsilon}^{m} \rightarrow Q^{m}$ strongly in $H$ and the simple inclusion $H \subset L^{1}(\mathcal{M})$, we have by passing to the limit on $\varepsilon$ that

$$
\left\langle H_{\varepsilon_{2}}\left(Q_{\varepsilon}^{m}-q_{s}\right), q^{b}-Q_{\varepsilon}^{m}\right\rangle \rightarrow\left\langle h_{Q^{m}}, q^{b}-Q^{m}\right\rangle \quad \text { for } q^{b} \in V .
$$

Noticing that $K_{\varepsilon_{2}}(r)=0$ for $r<0$ and (3.52), we have

$$
\left|\left(K_{\varepsilon_{2}}\left(q^{b}-q_{s}\right), 1\right)-\left(\left[q^{b}-q_{s}\right]^{+}, 1\right)\right|=\left|\left(K_{\varepsilon_{2}}\left(\left[q^{b}-q_{s}\right]^{+}\right)-\left[q^{b}-q_{s}\right]^{+}, 1\right)\right| \leq \frac{\varepsilon_{2}}{2}|\mathcal{M}|,
$$

which implies

$$
\left(K_{\varepsilon_{2}}\left(q^{b}-q_{s}\right), 1\right) \rightarrow\left(\left[q^{b}-q_{s}\right]^{+}, 1\right) \quad \text { as } \varepsilon_{2} \rightarrow 0+.
$$

To show the convergence of $\left(K_{\varepsilon_{2}}\left(Q_{\varepsilon}^{m}-q_{s}\right), 1\right)$ to $\left(\left[Q^{m}-q_{s}\right]^{+}, 1\right)$, we split the difference $\left(K_{\varepsilon_{2}}\left(Q_{\varepsilon}^{m}-q_{s}\right), 1\right)-\left(\left[Q^{m}-q_{s}\right]^{+}, 1\right)$ as the following sum:

$$
\left(\left(K_{\varepsilon_{2}}\left(Q_{\varepsilon}^{m}-q_{s}\right), 1\right)-\left(K_{\varepsilon_{2}}\left(Q^{m}-q_{s}\right), 1\right)\right)+\left(\left(K_{\varepsilon_{2}}\left(Q^{m}-q_{s}\right), 1\right)-\left(\left[Q^{m}-q_{s}\right]^{+}, 1\right)\right) .
$$

Copyright (c) by SIAM. Unauthorized reproduction of this article is prohibited. 
The second term in the above sum can be dealt with exactly as for (3.55). For the first term, we have by (3.51) and the Cauchy-Schwarz inequality that

$\left|\left(K_{\varepsilon_{2}}\left(Q_{\varepsilon}^{m}-q_{s}\right), 1\right)-\left(K_{\varepsilon_{2}}\left(Q^{m}-q_{s}\right), 1\right)\right| \leq\left(\left|\left(Q_{\varepsilon}^{m}-q_{s}\right)-\left(Q^{m}-q_{s}\right)\right|, 1\right) \leq \sqrt{|\mathcal{M}|}\left|Q_{\varepsilon}^{m}-Q^{m}\right|_{L^{2}}$, which implies, in view of the strong convergence of $Q_{\varepsilon}^{m}$ to $Q^{m}$ in $H$,

$$
\left(K_{\varepsilon_{2}}\left(Q_{\varepsilon}^{m}-q_{s}\right), 1\right) \rightarrow\left(\left[Q^{m}-q_{s}\right]^{+}, 1\right) \quad \text { as } \varepsilon \rightarrow(0+, 0+) .
$$

From (3.53)-(3.56), we conclude that

$$
\left(\left[q^{b}-q_{s}\right]^{+}, 1\right)-\left(\left[Q^{m}-q_{s}\right]^{+}, 1\right) \geq\left\langle h_{Q^{m}}, q^{b}-Q^{m}\right\rangle,
$$

which amounts to saying that $h_{Q^{m}} \in \mathcal{H}\left(Q^{m}-q_{s}\right)$.

Summarizing the above arguments, we have the following lemma.

Lemma 3.12. For each $m=1, \ldots, N$, there exists $\left(T^{m}, q^{m}\right) \in V \times \mathcal{K}$ which is a solution of $(3.3)-(3.4)$.

4. Convergence of the Euler scheme. In this section, we want to prove the convergence of the solutions of the Euler scheme (3.3)-(3.4) to the solutions of the system (2.23)-(2.25). We shall use the same conventions on subsequences and indices as in the last section, that is, the limit process in this part is $N \rightarrow+\infty$ or, equivalently, $k \rightarrow 0+$ and up to subsequences.

Due to the weak lower semicontinuity property of the norms, we know that for the limit functions $U^{m}$ which now have no dependence on $\varepsilon$, the bounds in Lemmas 3.5 and 3.9 are now valid with $U_{\varepsilon}^{m}$ replaced by the limit functions $U^{m}$.

For each fixed $k$ ( or $N$ ), we associate to the elements $U^{0}=U_{0}, U^{1}, U^{2}, \ldots, U^{N}$ the following approximate functions: $U_{k}=\left(T_{k}, q_{k}\right), \tilde{U}_{k}=\left(\tilde{T}_{k}, \tilde{q}_{k}\right)$, and $W_{k}=\left(\mathcal{T}_{k}, \mathcal{Q}_{k}\right)$, which are defined piecewise on $\left[0, t_{1}\right]$ and take values in the space $V^{2}$ :

$$
U_{k}(t)=U^{m}, \quad \tilde{U}_{k}(t)=U^{m-1} \quad \text { for } t \in[(m-1) k, m k), m=1,2, \ldots, N,
$$

and $W_{k}$ is the continuous function equal to $U^{m}$ at $m k$ and linear between $(m-1) k$ and $m k$.

4.1. A priori estimates. We start with a lemma which is essentially a rephrasing of the estimates in Lemmas 3.5 and 3.9 with $U_{\varepsilon}^{m}$ replaced by $U^{m}$.

LEMma 4.1. The functions $U_{k}, \tilde{U}_{k}, W_{k}$ remain in a bounded set of $L^{2}\left(0, t_{1} ; V\right) \cap$ $L^{\infty}\left(0, t_{1} ; H\right)$ as $k \rightarrow 0+$. The functions $\partial_{t} W_{k}$ form a bounded set in $L^{2}\left(0, t_{1} ; V^{*}\right)$, and $U_{k}-W_{k} \rightarrow 0$ in $L^{2}\left(0, t_{1} ; H\right)$ strongly as $k \rightarrow 0+$.

We continue with the following estimates.

Lemma 4.2. For the functions $U_{k}, \tilde{U}_{k}, W_{k}$ defined above, there holds

$$
\begin{aligned}
\left|U_{k}-W_{k}\right|_{L^{2}\left(0, t_{1} ; H\right)} & \leq C\left(\mathbf{u}, U_{0}, t_{1}\right) \sqrt{k}, \\
\left|U_{k}-\tilde{U}_{k}\right|_{L^{2}\left(0, t_{1} ; H\right)} & \leq C\left(\mathbf{u}, U_{0} t_{1}\right) \sqrt{k} .
\end{aligned}
$$

Proof. The estimate (4.2) is well known and proved in, e.g., Temam [27]; the estimate (4.3) is straightforward.

Now we define $\mathbf{u}_{k}:\left[0, t_{1}\right] \rightarrow \mathbf{V}$ as follows:

$$
\mathbf{u}_{k}(t)=\mathbf{u}^{m} \quad \text { for } t \in[(m-1) k, m k), m=1,2, \ldots, N .
$$

We have the following lemma. 
Lemma 4.3 (convergence of $\mathbf{u}_{k}$ ). For the functions $\mathbf{u}_{k}$ defined above, there holds

$$
\mathbf{u}_{k} \rightarrow \mathbf{u} \text { in } L^{r}\left(0, t_{1}, \mathbf{V}\right) \text { as } k \rightarrow 0+
$$

Proof. The map $\mathbf{u} \rightarrow \mathbf{u}_{k}$ is a linear averaging transformation. By (3.2) and reasoning similar to that in (3.39) (replacing 4 by $r$ ), we know that the map is bounded:

$$
\left|\mathbf{u}_{k}\right|_{L^{r}\left(0, t_{1} ; \mathbf{V}\right)} \leq|\mathbf{u}|_{L^{r}\left(0, t_{1} ; \mathbf{V}\right)} .
$$

Due to the above bound, we could show the conclusion of the lemma by a density argument. As the space $C^{1}\left(\left[0, t_{1}\right] ; \mathbf{V}\right)$ is dense in $L^{r}\left(0, t_{1} ; \mathbf{V}\right)$, the conclusion of Lemma 4.3 follows by considering $\mathbf{u} \in C^{1}\left(\left[0, t_{1}\right] ; \mathbf{V}\right)$.

For later use, we also define the linear averaging map for the test functions $U^{b}=$ $\left(T^{b}, q^{b}\right) \in L^{2}\left(0, t_{1} ; V\right)$ that we will use below; that is, we define $U_{k}^{b}:\left[0, t_{1}\right] \rightarrow V^{2}$ piecewise by

$$
U_{k}^{b}(t)=\frac{1}{k} \int_{(m-1) k}^{m k} U^{b}(t) d t \text { on }[(m-1) k, m k) .
$$

Similarly as in Lemma 4.3, we conclude that $U_{k}^{b} \rightarrow U^{b}$ strongly in $L^{2}\left(0, t_{1} ; V^{2}\right)$ as $k \rightarrow 0$. Moreover, if $q^{b} \in \mathcal{K}$ for a.e. $t \in\left[0, t_{1}\right]$, we have $q_{k}^{b} \in \mathcal{K}$ for all $t \in\left[0, t_{1}\right]$.

4.2. Passage to the limit $\boldsymbol{k} \rightarrow \mathbf{0}+$. To proceed, we reinterpret as follows the scheme (3.3)-(3.4) in terms of the functions $U_{k}=\left(T_{k}, q_{k}\right), \tilde{U}_{k}=\left(\tilde{T}_{k}, \tilde{q}_{k}\right), W_{k}=$ $\left(\mathcal{T}_{k}, \mathcal{Q}_{k}\right)$, and $U_{k}^{b}=\left(T_{k}^{b}, q_{k}^{b}\right)$ :

$$
\begin{array}{r}
\left\langle\partial_{t} \mathcal{T}_{k}, T_{k}^{b}\right\rangle+a_{T}\left(T_{k}, T_{k}^{b}\right)+b_{T}\left(\mathbf{u}_{k}, T_{k}, T_{k}^{b}\right)-d\left(\omega_{k}, \tilde{T}_{k}, T_{k}^{b}\right)-l_{T}\left(T_{k}^{b}\right) \\
=\left(\frac{1}{p}\left[\omega_{k}\right]^{-} h_{Q_{k}} \varphi\left(\tilde{T}_{k}\right), T_{k}^{b}\right), \\
\left\langle\partial_{t} \mathcal{Q}_{k}, q_{k}^{b}-q_{k}\right\rangle+a_{q}\left(q_{k}, q_{k}^{b}-q_{k}\right)+b_{q}\left(\mathbf{u}_{k}, q_{k}, q_{k}^{b}-q_{k}\right)-l_{q}\left(q_{k}^{b}-q_{k}\right) \\
\geq\left(-\frac{1}{p}\left[\omega_{k}\right]^{-} h_{Q_{k}} F\left(\tilde{T}_{k}\right), q_{k}^{b}-q_{k}\right),
\end{array}
$$

where $Q_{k}$ is either $\tilde{q}_{k}$ or $q_{k}$ corresponding to Scheme A or Scheme B, respectively. Furthermore, $h_{Q_{k}}$ is defined by $h_{Q_{k}}(t)=h_{Q^{m}}$ when $t \in[(m-1) k, m k)$. Here we have considered an arbitrary $q^{b} \in L^{2}\left(0, t_{1} ; \mathcal{K}\right)$, and $q_{k}^{b}$ defined as $\mathbf{u}_{k}$ belongs to $L^{2}\left(0, t_{1} ; \mathcal{K}\right)$, and the analogue of Lemma 4.3 holds (with $q_{k}^{b} \rightarrow q^{b}$ in $L^{2}\left(0, t_{1} ; V\right)$ ).

Due to Lemma 4.1, we have, up to subsequences, in the limit $k \rightarrow 0+$, that

$$
\begin{gathered}
U_{k}, \tilde{U}_{k} \rightarrow U=(T, q) \quad \text { weakly in } L^{2}\left(0, t_{1} ; V\right) \text { and weak-* in } L^{\infty}\left(0, t_{1} ; H\right), \\
W_{k} \rightarrow W=(\mathcal{T}, \mathcal{Q}) \quad \text { weakly in } L^{2}\left(0, t_{1} ; V\right) \text { and weak-* in } L^{\infty}\left(0, t_{1} ; H\right),
\end{gathered}
$$

and

$$
\partial_{t} W_{k} \rightarrow \partial_{t} W=\left(\partial_{t} \mathcal{T}, \partial_{t} \mathcal{Q}\right) \quad \text { weakly in } L^{2}\left(0, t_{1} ; V^{*}\right) .
$$

In view of Lemma 4.2 , we know that

$$
U=W .
$$

Now, we consider the inclusions $V \subset H \subset V^{*}$ and recall that the first inclusion is compact and the second inclusion is continuous. In view of (4.9) and (4.10), we conclude, by applying the Aubin-Lions compactness theorem, that

$$
W_{k} \rightarrow W \text { strongly in } L^{2}\left(0, t_{1} ; H\right) .
$$


By Lemma 4.2 again, we conclude that

$$
U_{k}, \tilde{U}_{k}, W_{k} \rightarrow U \text { strongly in } L^{2}\left(0, t_{1} ; H\right) .
$$

We are now in a position to pass to the limit in (4.7). To proceed, let us illustrate in advance that the limit function $q$ lies in $L^{2}\left(0, t_{1} ; \mathcal{K}\right)$. Indeed, regarded as a convex subset of $L^{2}\left(0, t_{1} ; V\right), L^{2}\left(0, t_{1} ; \mathcal{K}\right)$ is closed with respect to the strong topology induced by the $L^{2}\left(0, t_{1} ; V\right)$-norm. Therefore, it is also closed with respect to the weak topology. First, as $q_{k} \rightarrow q$ weakly in $L^{2}\left(0, t_{1} ; V\right)$ and $q_{k}^{b} \rightarrow q^{b}$ strongly in $L^{2}\left(0, t_{1} ; V\right)$, we know that $\gamma_{0}\left(q_{k}\right) \rightarrow \gamma_{0}(q)$ and $\gamma_{0}\left(q_{k}^{b}\right) \rightarrow \gamma_{0}\left(q^{b}\right)$ weakly in $L^{2}\left(0, t_{1} ; L^{2}(\partial \mathcal{M})\right)$, where $\gamma_{0}$ is the trace operator on $\partial \mathcal{M}$. Hence,

$$
\int_{0}^{t_{1}}\left(l_{q}\left(q_{k}^{b}-q_{k}\right)-l_{q}\left(q^{b}-q\right)\right) d t \rightarrow 0 \quad \text { as } k \rightarrow 0+.
$$

Second, we pass to the upper limit in $a_{q}\left(q_{k}, q_{k}^{b}-q_{k}\right)$ exactly as in (3.47), and we find

$$
\limsup \int_{0}^{t_{1}} a_{q}\left(q_{k}, q_{k}^{b}-q_{k}\right) d t \leq \int_{0}^{t_{1}} a_{q}\left(q, q^{b}-q\right) d t
$$

Third, we consider the convergence of the term $\int_{0}^{t_{1}}\left\langle\partial_{t} \mathcal{Q}_{k}, q_{k}^{b}-q_{k}\right\rangle d t$ which is the sum of $\int_{0}^{t_{1}}\left\langle\partial_{t} \mathcal{Q}_{k}, q_{k}^{b}-\mathcal{Q}_{k}\right\rangle d t$ and $\int_{0}^{t_{1}}\left\langle\partial_{t} \mathcal{Q}_{k}, \mathcal{Q}_{k}-q_{k}\right\rangle d t$. Using integration by parts, (4.10), and the lower semicontinuity of the norm, we write

$$
\begin{aligned}
\limsup \int_{0}^{t_{1}}\left\langle\partial_{t} \mathcal{Q}_{k}, q_{k}^{b}-\mathcal{Q}_{k}\right\rangle d t & =-\liminf \int_{0}^{t_{1}}\left\langle\partial_{t} \mathcal{Q}_{k}, \mathcal{Q}_{k}\right\rangle d t+\lim \int_{0}^{t_{1}}\left\langle\partial_{t} \mathcal{Q}_{k}, q_{k}^{b}\right\rangle d t \\
& =-\liminf \frac{1}{2}\left|\mathcal{Q}_{k}\left(t_{1}\right)\right|_{L^{2}}^{2}+\frac{1}{2}\left|q_{0}\right|_{L^{2}}^{2}+\int_{0}^{t_{1}}\left\langle\partial_{t} q, q^{b}\right\rangle d t \\
& \leq-\frac{1}{2}\left|q\left(t_{1}\right)\right|_{L^{2}}^{2}+\frac{1}{2}\left|q_{0}\right|_{L^{2}}^{2}+\int_{0}^{t_{1}}\left\langle\partial_{t} q, q^{b}\right\rangle d t \\
& =-\int_{0}^{t_{1}}\left\langle\partial_{t} q, q\right\rangle d t+\int_{0}^{t_{1}}\left\langle\partial_{t} q, q^{b}\right\rangle d t \\
& =\int_{0}^{t_{1}}\left\langle\partial_{t} q, q^{b}-q\right\rangle d t
\end{aligned}
$$

where we have used, in the second equality of (4.16), the observation

$$
\lim \int_{0}^{t_{1}}\left\langle\partial_{t} \mathcal{Q}_{k}, q_{k}^{b}\right\rangle d t \rightarrow \int_{0}^{t_{1}}\left\langle\partial_{t} q, q^{b}\right\rangle d t
$$

which follows from (4.10) and $q_{k}^{b} \rightarrow q^{b}$ in $L^{2}\left(0, t_{1} ; V\right)$.

A subtle point is the treatment of $\int_{0}^{t_{1}}\left\langle\partial_{t} \mathcal{Q}_{k}, \mathcal{Q}_{k}-q_{k}\right\rangle d t$. Though we have (4.10) (which implies in particular that $\partial_{t} \mathcal{Q}_{k}$ is bounded in $L^{2}\left(0, t_{1} ; V^{*}\right)$ ) and $\mathcal{Q}_{k}-q_{k} \rightarrow 0$ weakly in $L^{2}\left(0, t_{1} ; V\right)$, we cannot conclude that the limit of $\int_{0}^{t_{1}}\left\langle\partial_{t} \mathcal{Q}_{k}, \mathcal{Q}_{k}-q_{k}\right\rangle d t$ is 0 . Rather, we show, by the specific forms of $\mathcal{Q}_{k}$ and $q_{k}$, that

$$
\limsup \int_{0}^{t_{1}}\left\langle\partial_{t} \mathcal{Q}_{k}, \mathcal{Q}_{k}-q_{k}\right\rangle d t \leq 0 .
$$

Copyright $@$ by SIAM. Unauthorized reproduction of this article is prohibited. 
Indeed, noticing that $\partial_{t} \mathcal{Q}_{k}=\frac{q^{m}-q^{m-1}}{k}$ and $\mathcal{Q}_{k}-q_{k}=\frac{q^{m}-q^{m-1}}{k}(t-m k)$ on the subinterval $[(m-1) k, m k)$ of $\left[0, t_{1}\right]$, we have

$$
\begin{aligned}
\int_{0}^{t_{1}}\left\langle\partial_{t} \mathcal{Q}_{k}, \mathcal{Q}_{k}-q_{k}\right\rangle d t & =\sum_{m=1}^{N} \int_{(m-1) t}^{m t}\left\langle\partial_{t} \mathcal{Q}_{k}, \mathcal{Q}_{k}-q_{k}\right\rangle d t \\
& =\sum_{m=1}^{N} \int_{(m-1) t}^{m k}\left\langle\frac{q^{m}-q^{m-1}}{k}, \frac{q^{m}-q^{m-1}}{k}(t-m k)\right\rangle d t \\
& =\sum_{m=1}^{N} \int_{(m-1) t}^{m k} \frac{\left|q^{m}-q^{m-1}\right|_{L^{2}}^{2}}{k^{2}}(t-m k) d t \leq 0
\end{aligned}
$$

which implies (4.18). From (4.16) and (4.18), we can conclude that

$$
\limsup \int_{0}^{t_{1}}\left\langle\partial_{t} \mathcal{Q}_{k}, q_{k}^{b}-q_{k}\right\rangle d t \leq \int_{0}^{t_{1}}\left\langle\partial_{t} q, q^{b}-q\right\rangle d t .
$$

Fourth, we consider the convergence of the integral $\int_{0}^{t_{1}} b_{q}\left(\mathbf{u}_{k}, q_{k}, q_{k}^{b}-q_{k}\right) d t$.

We observe that $U_{k}, \tilde{U}_{k}$, and $W_{k}$ all lie in a bounded set of $L^{\infty}\left(0, t_{1} ; H\right)$ and converge to $U$ a.e. in $H$ for $t \in\left[0, t_{1}\right]$. By extraction of a subsequence this, together with (4.13) and the Lebesgue dominated convergence theorem, yields

$$
U_{k}, \tilde{U}_{k}, W_{k} \rightarrow U \text { strongly in } L^{p}\left(0, t_{1} ; H\right) \text { for any } p \in[1,+\infty) .
$$

As the functions $\mathbf{u}$ and $\mathbf{u}^{m}$ are divergence free, we have, using (2.19),

$$
\begin{aligned}
& \int_{0}^{t_{1}} b_{q}\left(\mathbf{u}_{k}, q_{k}, q_{k}^{b}-q_{k}\right)-b_{q}\left(\mathbf{u}, q, q^{b}-q\right) d t=\int_{0}^{t_{1}} b_{q}\left(\mathbf{u}_{k}, q_{k}, q_{k}^{b}\right)-b_{q}\left(\mathbf{u}, q, q^{b}\right) d t \\
= & \int_{0}^{t_{1}} b_{q}\left(\mathbf{u}_{k}-\mathbf{u}, q_{k}, q_{k}^{b}\right) d t+\int_{0}^{t_{1}} b_{q}\left(\mathbf{u}, q_{k}-q, q_{k}^{b}\right) d t+\int_{0}^{t_{1}} b_{q}\left(\mathbf{u}, q, q_{k}^{b}-q^{b}\right) d t \\
:= & \mathcal{I}_{1}+\mathcal{I}_{2}+\mathcal{I}_{3} .
\end{aligned}
$$

The terms $\mathcal{I}_{1}$ and $\mathcal{I}_{2}$ can be controlled by applying Lemma 2.1 and Hölder's inequality as follows, where $r^{*}>4$ satisfies $\frac{1}{r}+\frac{1}{r^{*}}=\frac{1}{4}$ :

$$
\begin{aligned}
\left|\mathcal{I}_{1}\right|+\left|\mathcal{I}_{2}\right| \leq & C \int_{0}^{t_{1}}\left|\mathbf{u}_{k}-\mathbf{u}\right|_{\mathbf{V}}\left|q_{k}\right|_{L^{2}}^{\frac{1}{2}}\left\|q_{k}\right\|\left\|^{\frac{1}{2}}\right\| q_{k}^{b}\left\|d t+C \int_{0}^{t_{1}}|\mathbf{u}|_{\mathbf{V}}\left|q_{k}-q\right|_{L^{2}}^{\frac{1}{2}}\right\| q_{k}-q\left\|^{\frac{1}{2}}\right\| q_{k}^{b} \| d t \\
\leq & C\left|\mathbf{u}_{k}-\mathbf{u}\right|_{L^{r}\left(0, t_{1} ; \mathbf{V}\right)}\left|q_{k}\right|_{L^{\frac{r^{*}}{2}}\left(0, t_{1} ; H\right)}^{\frac{1}{2}}\left|q_{k}\right|_{L^{2}\left(0, t_{1} ; V\right)}^{\frac{1}{2}}\left|q_{k}^{b}\right|_{L^{2}\left(0, t_{1} ; V\right)} \\
& +C|\mathbf{u}|_{L^{r}\left(0, t_{1} ; \mathbf{V}\right)}\left|q_{k}-q\right|_{L^{\frac{r^{*}}{2}}\left(0, t_{1} ; H\right)}\left|q_{k}-q\right|_{L^{2}\left(0, t_{1} ; V\right)}^{\frac{1}{2}}\left|q_{k}^{b}\right|_{L^{2}\left(0, t_{1} ; V\right)} .
\end{aligned}
$$

By applying Lemma 2.1 and noticing the strong convergence of $q_{k}^{b}$ to $q^{b}$ in $L^{2}\left(0, t_{1} ; V\right)$, we easily see that

$$
\left|\mathcal{I}_{3}\right| \rightarrow 0 \quad \text { as } k \rightarrow 0+.
$$

Using (4.8), (4.20), and Lemma 4.3, we conclude from (4.21)-(4.23) that

$$
\int_{0}^{t_{1}}\left[b_{q}\left(\mathbf{u}_{k}, q_{k}, q_{k}^{b}-q_{k}\right)-b_{q}\left(\mathbf{u}, q, q^{b}-q\right)\right] d t \rightarrow 0 .
$$

Copyright $@$ by SIAM. Unauthorized reproduction of this article is prohibited. 
Fifth, we consider the variational inequality involving $h_{Q_{k}}$. By (3.57) and the definitions of $h_{Q_{k}}$ and $q_{k}^{b}$, we have for every $q^{b} \in L^{2}\left(0, t_{1} ; V\right)$

$$
\left(\left[q_{k}^{b}-q_{s}\right]^{+}, 1\right)-\left(\left[Q_{k}-q_{s}\right]^{+}, 1\right) \geq\left\langle h_{Q_{k}}, q_{k}^{b}-Q_{k}\right\rangle \text { for a.e. } t \in\left[0, t_{1}\right],
$$

and integrating in $t$ from 0 to $t_{1}$, we find

$$
\int_{0}^{t_{1}}\left(\left[q_{k}^{b}-q_{s}\right]^{+}, 1\right) d t-\int_{0}^{t_{1}}\left(\left[Q_{k}-q_{s}\right]^{+}, 1\right) d t \geq \int_{0}^{t_{1}}\left\langle h_{Q_{k}}, q_{k}^{b}-Q_{k}\right\rangle d t .
$$

Up to a subsequence, we know that $h_{Q_{k}}$ converges to some limit $h_{q}$ weak-* in $L^{\infty}\left(\left[0, t_{1}\right] \times \mathcal{M}\right)$ and $0 \leq h_{q} \leq 1$ a.e. In view of the strong convergence of $q_{k}^{b}-Q_{k}$ to $q^{b}-q$ in $L^{2}\left(0, t_{1} ; H\right)$, we conclude that

$$
\int_{0}^{t_{1}}\left\langle h_{Q_{k}}, q_{k}^{b}-Q_{k}\right\rangle d t \rightarrow \int_{0}^{t_{1}}\left\langle h_{q}, q^{b}-q\right\rangle d t
$$

Moreover, we have

$$
\begin{aligned}
\left|\int_{0}^{t_{1}}\left(\left[Q_{k}-q_{s}\right]^{+}-\left[q-q_{s}\right]^{+}, 1\right) d t\right| & \leq \int_{0}^{t_{1}}\left|Q_{k}-q\right|_{L^{2}}|1|_{L^{2}} d t \\
& \leq\left|Q_{k}-q\right|_{L^{2}\left(0, t_{1} ; H\right)} \sqrt{|\mathcal{M}| t_{1}} \rightarrow 0
\end{aligned}
$$

in view of the strong convergence of $Q_{k}$ to $q$ in $L^{2}\left(0, t_{1} ; H\right)$.

Similarly as in (4.28), we could conclude the following convergence by noticing the strong convergence of $q_{k}^{b}$ to $q^{b}$ in $L^{2}\left(0, t_{1} ; V\right)$ :

$$
\int_{0}^{t_{1}}\left(\left[q_{k}^{b}-q_{s}\right]^{+}, 1\right) d t \rightarrow \int_{0}^{t_{1}}\left(\left[q^{b}-q_{s}\right]^{+}, 1\right) d t .
$$

In view of (4.27)-(4.29), we conclude that the following inequality holds after passing to the limit in (4.26):

$$
\int_{0}^{t_{1}}\left(\left[q^{b}-q_{s}\right]^{+}, 1\right) d t-\int_{0}^{t_{1}}\left(\left[q-q_{s}\right]^{+}, 1\right) d t \geq \int_{0}^{t_{1}}\left\langle h_{q}, q^{b}-q\right\rangle d t
$$

for every $q^{b} \in L^{2}\left(0, t_{1} ; V\right)$. This inequality implies that

$$
\left(\left[q^{b}-q_{s}\right]^{+}, 1\right)-\left(\left[q-q_{s}\right]^{+}, 1\right) \geq\left\langle h_{q}, q^{b}-q\right\rangle \text { for a.e. } t \in\left[0, t_{1}\right],
$$

for every $q^{b} \in L^{2}\left(0, t_{1} ; V\right)$, and thus $h_{q} \in \mathcal{H}\left(q-q_{s}\right)$.

Sixth, we take care of the convergence of $\int_{0}^{t_{1}}\left(-\frac{1}{p}\left[\omega_{k}\right]^{-} h_{Q_{k}} F\left(\tilde{T}_{k}\right), q_{k}^{b}-q_{k}\right) d t$. To proceed, we write the difference

$$
\int_{0}^{t_{1}}\left(-\frac{1}{p}\left[\omega_{k}\right]^{-} h_{Q_{k}} F\left(\tilde{T}_{k}\right), q_{k}^{b}-q_{k}\right) d t-\int_{0}^{t_{1}}\left(-\frac{1}{p}[\omega]^{-} h_{q} F(T), q^{b}-q\right) d t
$$

as the sum of the following two terms:

$$
\begin{gathered}
\int_{0}^{t_{1}}\left(-\frac{1}{p}\left(\left[\omega_{k}\right]^{-}-[\omega]^{-}\right) h_{Q_{k}} F\left(\tilde{T}_{k}\right), q_{k}^{b}-q_{k}\right) d t \\
\int_{0}^{t_{1}}\left(-\frac{1}{p}[\omega]^{-} h_{Q_{k}} F\left(\tilde{T}_{k}\right), q_{k}^{b}-q_{k}\right)-\left(-\frac{1}{p}[\omega]^{-} h_{q} F(T), q^{b}-q\right) d t
\end{gathered}
$$

Copyright (c) by SIAM. Unauthorized reproduction of this article is prohibited. 
The first term above tends to 0 due to the following inequality:

$$
\int_{0}^{t_{1}}\left(-\frac{1}{p}\left(\left[\omega_{k}\right]^{-}-[\omega]^{-}\right) h_{Q_{k}} F\left(\tilde{T}_{k}\right), q_{k}^{b}-q_{k}\right) d t \leq C\left|\omega_{k}-\omega\right|_{L^{2}\left(0, t_{1} ; V\right)}\left|q_{k}^{b}-q_{k}\right|_{L^{2}\left(0, t_{1} ; H\right)} .
$$

Noticing that $h_{Q_{k}} \rightarrow h_{q}$ weak- $*$ in $L^{\infty}\left(\left[0, t_{1}\right] \times \mathcal{M}\right)$ and $F$ is Lipschitz continuous, we know that $h_{Q_{k}} F\left(\tilde{T}_{k}\right) \rightarrow h_{q} F(T)$ weakly in $L^{2}\left(0, t_{1} ; H\right)$. Further, noticing that $q_{k}^{b}-q_{k} \rightarrow q^{b}-q$ strongly in $L^{2}\left(0, t_{1} ; H\right)$, we conclude that the second term above also tends to zero.

By now, we have shown that

$$
\int_{0}^{t_{1}}\left(-\frac{1}{p}\left[\omega_{k}\right]^{-} h_{Q_{k}} F\left(\tilde{T}_{k}\right), q_{k}^{b}-q_{k}\right) d t \rightarrow \int_{0}^{t_{1}}\left(-\frac{1}{p}[\omega]^{-} h_{q} F(T), q^{b}-q\right) d t
$$

Finally, we observe that $W_{k}(0)=U_{0}$, which follows from the definition of $W_{k}$.

In view of (4.14), (4.15), (4.19), (4.24), (4.31), and (4.32), we have shown that the inequalities $(2.24)$ and $(2.25)$ hold for $(T, q)$. The task of showing that (2.23) holds for $(T, q)$ is similar to or significantly easier than that for $(2.24)$. We omit the details here.

To sum up, we have proved the following theorem.

THEOREM 4.4. Given $T_{0}, q_{0} \in H$ with $0 \leq q_{0} \leq 1$ a.e. in $\mathcal{M}$, the functions $U_{k}, \tilde{U}_{k}$, and $W_{k}$ associated with the Euler scheme (3.3)-(3.4) contain a subsequence $k \rightarrow 0$ which converges to a solution $U$ of the system (2.23)-(2.25) in the sense of (4.8)-(4.13).

Remark 4.5. Theorem 4.4 implies the existence of a solution for the system (2.23)(2.25) which was proved in [31] by a different method.

\section{REFERENCES}

[1] A. Bousquet, M. Coti Zelati, And R. Temam, Phase transition models in atmospheric dynamics, Milan J. Math., 82 (2014), pp. 99-128, https://doi.org/10.1007/ s00032-014-0213-y.

[2] H. Brézis, Problèmes unilatéraux, J. Math. Pures Appl. (9), 51 (1972), pp. 1-168.

[3] C. CaO And E. S. Titi, Global well-posedness of the three-dimensional viscous primitive equations of large scale ocean and atmosphere dynamics, Ann. of Math. (2), 166 (2007), pp. 245-267, https://doi.org/10.4007/annals.2007.166.245.

[4] M. Coti Zelati, M. Frémond, R. Temam, and J. Tribbia, The equations of the atmosphere with humidity and saturation: Uniqueness and physical bounds, Phys. D, 264 (2013), pp. 49-65, https://doi.org/10.1016/j.physd.2013.08.007.

[5] M. Coti Zelati, A. Huang, I. Kukavica, R. Temam, and M. Ziane, The primitive equations of the atmosphere in presence of vapour saturation, Nonlinearity, 28 (2015), pp. 625-668, https://doi.org/10.1088/0951-7715/28/3/625.

[6] J.-I. DíAz, Mathematical analysis of some diffusive energy balance models in climatology, in Mathematics, Climate and Environment, RMA Res. Notes Appl. Math. 27, J.-I. Díaz and J.-L. Lions, eds., Masson, Paris, 1993, pp. 28-56.

[7] J. I. Díaz AND L. Tello, A nonlinear parabolic problem on a Riemannian manifold without boundary arising in climatology, Collect. Math., 50 (1999), pp. 19-51.

[8] G. Duvaut and J.-L. Lions, Inequalities in Mechanics and Physics, translated from the French by C. W. John, Springer-Verlag, Berlin, New York, 1976.

[9] I. Ekeland And R. Temam, Convex Analysis and Variational Problems, Classics Appl. Math. 28, SIAM, Philadelphia, 1999, https://doi.org/10.1137/1.9781611971088.

[10] E. Feireisl and J. Norbury, Some existence, uniqueness and nonuniqueness theorems for solutions of parabolic equations with discontinuous nonlinearities, Proc. Roy. Soc. Edinburgh Sect. A, 119 (1991), pp. 1-17, https://doi.org/10.1017/S0308210500028262.

[11] M. Frémond, Phase Change in Mechanics, Springer-Verlag, Berlin, Heidelberg, 2012. 
[12] R. Gianni And J. Hulshof, The semilinear heat equation with a Heaviside source term, European J. Appl. Math., 3 (1992), pp. 369-379, https://doi.org/10.1017/S0956792500000917.

[13] A. E. Gill, Atmosphere-Ocean Dynamics, Int. Geophys. Ser. 30, Academic Press, San Diego, 1982.

[14] W. W. Grabowski and P. K. Smolarkiewicz, A multiscale anelastic model for meteorological research, Mon. Weather Rev., 130 (2002), pp. 939-956, https://doi.org/10.1175/ 1520-0493(2002)130<0939:AMAMFM>2.0.CO;2.

[15] B. Guo AND D. HuAng, Existence of weak solutions and trajectory attractors for the moist atmospheric equations in geophysics, J. Math. Phys., 47 (2006), 083508, https://doi.org/ $10.1063 / 1.2245207$.

[16] B. Guo And D. Huang, Existence of the universal attractor for the 3-D viscous primitive equations of large-scale moist atmosphere, J. Differential Equations, 251 (2011), pp. 457491, https://doi.org/10.1016/j.jde.2011.05.010.

[17] G. J. Haltiner, Numerical Weather Prediction, John Wiley \& Sons, New York, 1971.

[18] G. J. Haltiner and R. T. Williams, Numerical Prediction and Dynamic Meteorology, John Wiley \& Sons, New York, 1980.

[19] D. Kinderlehrer and G. Stampacchia, An Introduction to Variational Inequalities and Their Applications, Academic Press, Harcourt Brace Jovanovich, New York, London, 1980. Reprinted as Classics in Appl. Math. 31, SIAM, Philadelphia, 2000, https://doi.org/10. $1137 / 1.9780898719451$.

[20] J.-L. Lions, Quelques méthodes de résolution des problèmes aux limites non linéaires, Dunod, Paris, 1969, reprinted in 2002.

[21] J.-Lions, R. Temam, and S. H. Wang, New formulations of the primitive equations of atmosphere and applications, Nonlinearity, 5 (1992), pp. 237-288, https://doi.org/10.1088/ 0951-7715/5/2/001.

[22] J. Pedlosky, Geophysical Fluid Dynamics, Springer-Verlag, New York, 1987.

[23] M. Petcu, R. Temam, And M. Ziane, Some mathematical problems in geophysical fluid dynamics, in Handbook of Numerical Analysis, Vol. 14, Special Volume: Computational Methods for the Atmosphere and the Oceans, Elsevier/North-Holland, Amsterdam, 2009, pp. $577-750$.

[24] R. T. Rockafellar, Convex Analysis, Princeton University Press, Princeton, NJ, 1997.

[25] R. R. Rogers and M. K. Yau, A Short Course in Cloud Physics, Pergamon Press, Oxford, New York, 1989

[26] R. Temam, Navier-Stokes Equations and Nonlinear Functional Analysis, 2nd ed., CBMS-NSF Regional Conf. Ser. in Appl. Math. 66, SIAM, Philadelphia, 1995, https://doi.org/10.1137/ 1.9781611970050 .

[27] R. Temam, Navier-Stokes Equations: Theory and Numerical Analysis, AMS Chelsea Publishing, Providence, RI, 2001.

[28] R. Temam and J. TribBia, Uniqueness of solutions for moist advection problems, Quart. J. Royal Meteorol. Soc., 140 (2014), pp. 1315-1318, https://doi.org/10.1002/qj.2217.

[29] R. Temam and J. Tribbia, The equations of the moist advection: A unilateral problem, Quart. J. Royal Meteorol. Soc., 142 (2016), pp. 143-146, https://doi.org/10.1002/qj.2638.

[30] R. Temam And X. WAng, Approximation of the equations of the humid atmosphere with saturation, in Proceedings of the 27th IFIP TC7 Conference 2015, Antibes, France, to appear.

[31] R. TEmam And K. Wu, Formulation of the equations of the humid atmosphere in the context of variational inequalities, J. Funct. Anal., 269 (2015), pp. 2187-2221, https://doi.org/10. 1016/j.jfa.2015.02.010.

[32] F. Tone And D. Wirosoetisno, On the long-time stability of the implicit Euler scheme for the two-dimensional Navier-Stokes equations, SIAM J. Numer. Anal., 44 (2006), pp. 29-40, https://doi.org/10.1137/040618527.

Copyright (c) by SIAM. Unauthorized reproduction of this article is prohibited. 